\title{
Zur Mikromorphologie und Genese lessivierter Böden im Jungmoränengebiet Schleswig-Holsteins und Mecklenburg-Vorpommern s
}

\author{
Peter Kühn, Peter Janetzko \& Dietmar Schröder*)
}

\begin{abstract}
Kühn, P., Janetzko , P. \& Schröder, D. (2002): Zur Mikromorphologie und Genese lessivierter Böden im Jungmoränengebiet Schleswig-Holsteins und Mecklenburg-Vorpommerns. - Eiszeitalter und Gegenwart, 51: 74-92; Hannover 2002.
\end{abstract}

Keywords: micromorphology, soil genesis, Luvisols, late glacial

Kurzfassung: In der deutschen Bodenkunde besagt die allgemein anerkannte Hypothese zur Lessivégenese, dass Lessivés auf weichseleiszeitlichen Sedimenten im norddeutschen Tiefland ausschließlich im Holozän entstanden seien, mit einer Hauptphase der Tonverlagerung im Atlantikum. Vornehmlich systematisch-mikromorphologische Untersuchungen an Braunerde-Lessivés aus Decksand über Geschiebelehm im norddeutschen Tiefland führen zu einer verbesserten Vorstellung über die chronologische Abfolge pedogenetischer Prozesse seit dem Beginn des Spätglazials. Es wird nachgewiesen, dass sowohl im Spätglazial, als auch im Holozän Tonverlagerung stattfand. Durch in Bt-Schmitzen inkorporierte Toncutane ist eine spätglaziale Tonverlagerung belegt, da die Entstehung dieser Schmitzen auf periglaziale Umlagerungsprozesse zurückgeführt werden kann. Ungestörte Toncutane neben Toncutanfragmenten zeigen nachfolgende holozäne Tonverlagerung an

\section{[Micromorphology and Genesis of Luvisols in the Young Moraine Area of Schleswig-Holstein and Mecklenburg-Vorpommern]}

\begin{abstract}
In German soil science the widely accepted hypothesis on Luvisol-formation states that Luvisols of Weichselian sediments are purely Holocene soils with a main formation phase during the Atlantic. Although controversially discussed since the sixties, the genesis of widespread albi-cambic Luvisols, with a simplified horizon sequence $\mathrm{Ah}, \mathrm{Ap} / \mathrm{Bw} / \mathrm{E}(/ \mathrm{E}+\mathrm{Bt}) / 2 \mathrm{Bt} / \mathrm{C}$, developed on the flat and slightly undulating till plains of the north German young moraine area, is not yet clarified. Especially systematic micromorphological analysis combined with (pedo-)stratigraphical work led to an improved understanding of the chronological order of soil forming processes since the beginning of the
\end{abstract}

\footnotetext{
*)Anschriften der Verfasser: Dipl. Geogr. P. KüHN, Universität Greifswald, Geogr. Inst., Jahnstr. 16, 17487 Greifswald. Dr. P. JANETZKo, LANU Schleswig-Holstein, 24220 Flintbek. Prof. Dr. D. Schröder, Universität Trier, FB VI, Abt. Bodenkunde, 54286 Trier.
}

Late Glacial. Fragments of clay coatings in periglacial formed Bt-streaks (occurring in $\mathrm{E}$ - and $\mathrm{E}+\mathrm{Bt}$-horizons) prove clay illuviation in the Late Glacial. Undisrupted clay coatings next to fragments of clay coatings prove a subsequent holocene clay illuviation. Contrary to the widely accepted soil-genetic hypothesis, clay illuviation is a pedogenic process occurring at least in warmer phases of the Late Glacial, together with the already accepted processes of decalcification, organic matter accumulation (bAh-horizons), podzolization and silicate weathering (bBw-horizons) on sites not influenced by groundwater.

\section{Einführung}

Im Jungmoränengebiet Norddeutschlands kommen auf flachwelligen bis ebenen Grundmoränenflächen als Leitböden lessivierte Böden vor. Typischerweise sind die oberen $5 \mathrm{dm}$ verbraunt, unter Wald meist flachgründig podsoliert. In der Regel beginnt unter einem 1-2 dm mächtigen Ael-Horizont in 6-8 dm Tiefe ein Tonanreicherungshorizont mit mehr oder minder starker Pseudovergleyung (JANETzKo \& Schmidt 1996). Eine vereinfachte typische Horizontabfolge dieser Leitböden ist Ah/ $\mathrm{Bv} / \mathrm{II}(\mathrm{Sw}-) \mathrm{A}(\mathrm{e}) \mathrm{l} /(\mathrm{Sw}-) \mathrm{A}(\mathrm{e}) \mathrm{l}+(\mathrm{Sd}-) \mathrm{Bt} /(\mathrm{Sd}-) \mathrm{Bt} / \mathrm{Cv}$. Die Braunerde ist zumeist im (Geschiebe-)Decksand ausgebildet, der Lessivé im liegenden Geschiebelehm.

Zur Decksandgenese, die nicht Gegenstand dieser Untersuchung ist, sei hier nur so viel gesagt, dass wir mit BusSEMER (1994) eine vorwiegend periglaziale Entstehung des Decksandes durch solimixtive Prozesse annehmen, jedoch die von HeLBIG (1999) vermutete holozäne Weiterprägung durch Tonauswaschung als Teilprozess der Decksandgenese nicht ausschließen.

Seit Jahrzehnten wird die Genese dieser Bodenprofile kontrovers diskutiert, ohne dass eine einheitliche Vorstellung dazu entstanden wäre. Dies schlägt sich auch in der unterschiedlichen Klassifikation dieser Profile mit o.g. Horizontabfolge durch die jeweiligen Bearbeiter nieder. So werden Profile ohne starken Hydromorphieeinfluß entweder als Braunerde-Lessivé, entsprechend der allgemein anerkannten Sichtweise einer rein holozä- 
nen Genese (z.B. REUTER 1990), oder als Braunerde mit (teil-)reliktischem Lessivé klassifiziert, entsprechend der bodengenetischen Vorstellung einer spätglazialen Genese des Lessivés im liegenden Geschiebelehm und der holozänen Braunerdebildung im jungdryaszeitlichen (Geschiebe-)Decksand im Hangenden (z.B. JANETZKO 1996).

Die Hypothese einer rein holozänen Pedogenese der Lessivés setzte sich als Lehrmeinung in Deutschland durch und wird in den einschlägigen bodenkundlichen Lehrbüchern vertreten. Dennoch wurden in den letzten Jahren einige stichhaltige Befunde zur spätglazialen Formung und holozänen Weiterbildung von Lessivés vorgestellt (z.B. KeMr et al. 1998, KüHN \& Kösel 2000, Rose et al. 2000, VAN VLIET-LANOË et al. 1992).

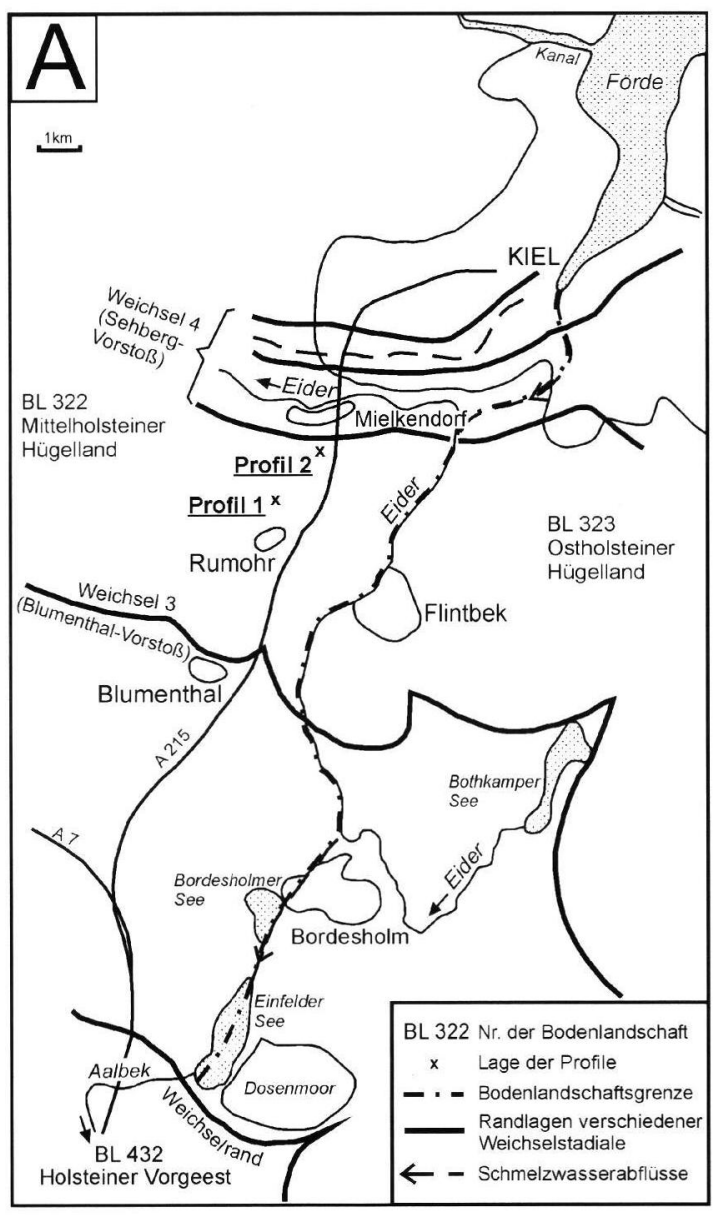

In den untersuchten Profilen können im Wesentlichen fünf pedogene Vorgänge festgestellt werden: Entkalkung - Verbraunung - Tonneubildung - Lessivierung - Pseudovergleyung. Daher stellt sich die Frage nach dem zeitlichen Ablauf dieser Prozesse, der anhand von vornehmlich mikromorphologischen Untersuchungen an drei ausgewählten Profilen mit unterschiedlicher Horizontausprägung beispielhaft vorgestellt wird.

\section{Methoden}

Die Korngrößenanalyse erfolgte nach kombinierter Sieb-Pipettmethode nach KÖHN (ScHLICHTING et al. 1995). Die bodenchemischen Daten wurden ebenfalls nach SCHLichting et al. (1995) ermittelt. Für die mikromorphologischen Untersuchungen

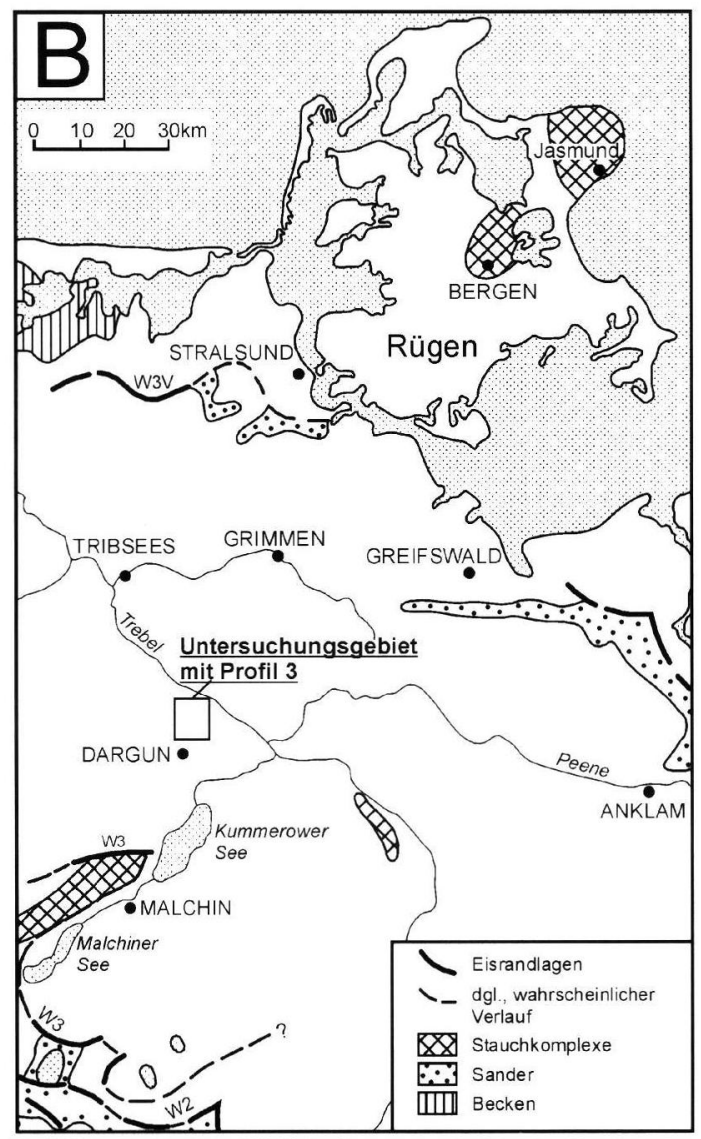

W3V V Velgaster Randlage, W3. Verbreitungsgrenze der Grundmoräne des Mecklenburger Vorstoßes, $z$.T. Rosenthaler Randlage, W2: Pommersche Hauptrandlage

Abb. 1: Lage des Untersuchungsraumes in Schleswig-Holstein (A) und in Mecklenburg Vorpommern (B); (B) stark vereinfacht aus RÜHBERG et al. (1995).

Fig. 1: Maps showing location of soil profiles in Schleswig-Holstein (A) and Mecklenburg-Vorpommern (B); (B) is simplified from RÜHBERG et al. (1995). 
wurden mit umgebauten Kubiëna-Kästchen (4,5 x $2,5 \times 2,5 \mathrm{~cm}$ bzw. $10 \times 6 \times 4 \mathrm{~cm})$ ungestörte orientierte Proben entnommen. Die Dünnschliffe wurden von der Firma T. BECKMANN hergestellt. Die Analyse der Dünnschliffe erfolgte vor allem nach Bullock et al. (1985) und STOOPs (1999). Im folgenden wird die Übersetzung des im Angloamerikanischen gebräuchlichen neutralen Wortes dusty (staubig) als adjektivische Kennzeichnung für Toncutane (clay coatings) und Porenverfüllungen (infillings) verwendet, die mit opaken Körnchen durchsetzt sind (z.B. Taf. 2). Das vor allem in der deutschen Mikromorphologie verbreitete Wort Granulierung wird hier nicht benutzt, da es meist mit dem von KuBIËNA (1986: 96) beschriebenen Alterungsprozess der Toncutane verbunden wird. Diese Granulierung soll nach SMOLIKOVA (1968) durch unpeptisierte Fe-Hydroxide hervorgerufen sein, während FELIX-HenNingsEn (1979: 48) von Schwermineralen in Grobton-Größe spricht. Die stoffliche Zusammensetzung dieser Körnchen ist noch nicht geklärt, ebenso wenig deren Genese. Denkbar ist auch eine einfache Verlagerung dieser Körnchen mit dem eingewaschenen Ton oder eine Entstehung während der Verlagerung (synilluvial), so dass nicht immer ein $\mathrm{Al}-$ terungsprozess für die staubige Erscheinung von Toncutanen verantwortlich wäre.

Toncutane mit einer Dicke von über $200 \mu \mathrm{m}$ werden zu den Porenverfüllungen (infillings) als SiltTon-Verfüllungen gezählt, um dem quantitativen Unterschied zu geringmächtigeren Toncutanen gerecht zu werden.

\section{Physisch-geographische Einordnung}

Die untersuchten Profile in Schleswig-Holstein befinden sich zwischen zwei Eisrandlagen der Weichselvereisung (siehe Abb. 1A). Das Klima des Untersuchungsraumes dürften die Werte der Klimastation Kiel (Daten des Wetteramtes Schleswig, langjähriges Mittel 1961-90) widerspiegeln; Jahresmittelwerte: $8,5^{\circ} \mathrm{C}\left(\operatorname{Jan}+0,7^{\circ} \mathrm{C}\right.$, Juli $\left.16,3^{\circ} \mathrm{C}\right)$ u. 783 $\mathrm{mm}$ (Sommerhalbjahr: $412 \mathrm{~mm}$ ).

Profil 1 befindet sich auf flachwelliger Grundmoräne zwischen der deutlich ausgeprägten Eisrandlage des Blumenthal-Vorstoßes und der Eisrandlage des jüngeren Sehberg-Vorstoßes. Nach STEPHAN (1995) ist ein Ende der Eisaktivität zwischen 14000 und 13000 BP anzunehmen. Die eher flachwellige Grundmoränenlandschaft bei Profil 1 (Rumohrholz) ist durch Pseudogleye geprägt. Die z.T. sehr unterschiedlichen Bodenformen mit oder ohne Staunässe beruhen auf den nach Mächtig- keit und Ausprägung verschiedenen Decksedimenten der Moräne. Diese sind teils aus solimixtiver Umlagerung unmittelbar aus der Moräne (typischer Geschiebedecksand), teils als solifluidales Umlagerungsprodukt, teils aus Schneeschmelzwässern (niveofluvial, z.B. Talsand) oder durch äolische Umlagerungen (Flugsand/Dünen) entstanden. Neben der Pseudovergleyung und Lessivierung im Geschiebelehm bestimmen deshalb Verbraunung und Podsolierung in den hangenden sandigen Deckschichten die Pedogenese (vgl. BÜK 200 Bl. CC 2326 Lübeck).

Profil 2 nahe einer Randlage des jüngeren Sehberg-Vorstoßes liegt in einer durch Kuppen und Toteislöcher (Sölle) geprägten (Ablations-)Moränenlandschaft. Zu den Bodenformen des Rumohrholzes gesellen sich hier aufgrund der vorhandenen Toteislöcher und Reste einer Schmelzwasser-Sanddecke noch Niedermoor, Kolluvisol und staunässefreie Böden.

Profil 3 liegt nordöstlich von Dargun auf flachwelliger Grundmoräne des Mecklenburger Vorstoßes nördlich der Pommerschen Eisrandlage (siehe Abb. 1B). Die etwas kontinentalere Prägung des Klimas dieses Untersuchungsgebietes zeigen die Daten der Klimastation Dargun (Klimatologische Normalwerte der DDR 1961: 1901-1950): Jahresmittelwerte: $8,0^{\circ} \mathrm{C}$ (Jan $-0,7^{\circ} \mathrm{C}$, Juli $17,4^{\circ} \mathrm{C}$ ) u. $598 \mathrm{~mm}$ (Sommerhalbjahr: $302 \mathrm{~mm}$ ).

Die Leitbodenform auf den Grundmoränenplatten nördlich von Dargun bei Groß Methling ist die Lessivé-Braunerde aus Decksand über Geschiebemergel, die manchmal schwach hydromorph beeinflusst ist. Daneben sind, durch Beakkerung verursacht, auf den flachen Kuppen häufig Pararendzinen und an Unterhängen Kolluvien verbreitet (SCHNEIDER \& KÜHN 2000). Da nach GÖRSDORF \& KAISER (2001) sich eine Eisfreiwerdung der Pommerschen Bucht um 14000 BP abzuzeichnen beginnt, kann dieses als Minimumalter der Enteisung für die Grundmoränenplatten des südlich gelegenen Festlandes gelten.

Bei Korrelation der Blumenthal-Eisrandlage mit der Pommerschen Eisrandlage und des SehbergVorstoßes mit dem Mecklenburger Vorstoß (STEPHAN 1995) ist davon auszugehen, dass beide Untersuchungsgebiete mindestens 3000-4000 Jahre den klimatischen Bedingungen des Spätglazials ausgesetzt waren.

\section{Ergebnisse}

Beispielhaft sollen hier drei typisch ausgebildete lessivierte Böden vorgestellt werden, die das Bo- 
Tab. 1: Analysedaten Profil 1.

Tab. 1: Grain size distribution and selected soil chemical data of soil profile 1.

\section{Profil 1: Rumohrholz 1, Wald}

\begin{tabular}{|c|c|c|c|c|c|c|c|c|c|c|c|c|c|c|c|}
\hline Horizont & Tiefe & P.-tiefe & $\mathrm{T}$ & $\mathrm{fU}$ & $\mathrm{mU}$ & $\mathrm{gU}$ & fS & $\mathrm{mS}$ & $\mathrm{gS}$ & $\mathrm{S}$ & $\mathrm{U}$ & $\mathrm{T}$ & B.-art & $\mathrm{pH}$ & $\mathrm{CaCO}_{3}$ \\
\hline & {$[\mathrm{cm}]$} & {$[\mathrm{cm}]$} & \multicolumn{10}{|c|}{$[\%]$} & & {$\left[\mathrm{CaCl}_{2}\right]$} & {$[\%]$} \\
\hline Aeh & -5 & $0-5$ & 8,3 & 2,2 & 5,5 & 10,1 & 19,0 & 41,5 & 13,3 & 74 & 18 & 8 & Sl3 & 3,0 & - \\
\hline Bsv & -14 & $8-13$ & 7,9 & 2,2 & 5,9 & 10,7 & 22,9 & 38,7 & 11,8 & 73 & 19 & 8 & S12 & 3,3 & - \\
\hline \multirow[t]{2}{*}{ II Bv } & -38 & $15-20$ & 2,9 & 1,3 & 2,5 & 14,2 & 50,9 & 23,1 & 5,0 & 79 & 18 & 3 & $\mathrm{Su} 2$ & 4,5 & - \\
\hline & & $30-35$ & 5,8 & 2,2 & 4,6 & 10,5 & 24,2 & 40,2 & 12,5 & 77 & 17 & 6 & $\mathrm{~S} 12$ & 4,3 & - \\
\hline $\mathrm{Bv}-\mathrm{lCv}$ & -53 & $40-45$ & 4,3 & 0,9 & 3,1 & 7,1 & 20,5 & 40,5 & 23,6 & 85 & 11 & 4 & $\mathrm{Su} 2$ & 4,4 & - \\
\hline III Bv-Sw & -70 & $55-60$ & 3,9 & 0,6 & 0,6 & 2,2 & 11,9 & 65,5 & 15,2 & 93 & 3 & 4 & $\mathrm{mS}$ & 4,3 & - \\
\hline \multirow[t]{2}{*}{$\mathrm{Sw}$} & -94 & $75-80$ & 3,8 & 1,7 & 3,7 & 4,2 & 8,1 & 36,1 & 42,3 & 87 & 10 & 4 & $\mathrm{mS}$ & 4,1 & - \\
\hline & & $85-90$ & 3,0 & 0,5 & 2,7 & 3,0 & 5,1 & 43,2 & 42,6 & 91 & 6 & 3 & $\mathrm{mS}$ & 4,1 & - \\
\hline \multirow[t]{2}{*}{ IV (Bt-)Sd } & -165 & $120-125$ & 20,7 & 6,1 & 11,3 & 13,8 & 28,6 & 16,6 & 3,0 & 48 & 31 & 21 & Ls3 & 5,8 & - \\
\hline & & $150-160$ & 19,5 & 5,4 & 10,9 & 13,8 & 24,5 & 19,0 & 6,9 & 50 & 30 & 20 & Ls3 & 7,1 & 0,5 \\
\hline eSd-lCv & $-180+$ & $190-195$ & 17,4 & 7,3 & 13,4 & 14,1 & 24,9 & 16,8 & 6,2 & 48 & 35 & 17 & Ls3 & 7,4 & 16,9 \\
\hline
\end{tabular}

denmosaik als Leitböden der flachwelligen bis ebenen und teilweise auch kuppigen Grundmoränenlandschaft prägen.

\subsection{Profil 1 (Rumohrholz 1)}

Das erste Profil vertritt lessivierte Böden mit einem bei etwa $100 \mathrm{~cm}$ unter GOF beginnenden Bt-Horizont. Nach feldbodenkundlicher Aufnahme konnte allerdings nicht mit eindeutiger Sicherheit ein Bt-Horizont nachgewiesen werden. Da in $120 \mathrm{~cm}$ Tiefe ein Schichtwechsel mit starker Zunahme des Tongehaltes vorliegt und Toncutane im Gelände nicht zweifelsfrei identifiziert werden konnten, wurde dieser Boden zunächst als Pseudogley-Braunerde klassifiziert. Charakteristisch für fast alle in den Untersuchungsgebieten unter Wald vorkommenden Braunerden ist die schwache Podsolierung.

\subsubsection{Analysedaten}

Besonders hervorzuheben ist die Schichtgrenze zwischen III Sw und IV Bt-Sd, die anhand der unterschiedlichen Verteilung der Sandgehalte leicht nachzuvollziehen ist und eine alleinige Erhöhung

Tab. 2: Ausgewählte mikromorphologische Hauptmerkmale von Profil 1. Klassifizierung ohne Quantifizierung nach Präsenz (Kreuz) oder Absenz (kein Kreuz). Zahlen in Klammern: mittlere Probenametiefe.

Tab. 2: Selected main micromorphological characteristics. The micromorphological property is shown by presence (cross) or absence (no cross). Numbers in brackets: average depth of sampling.

\begin{tabular}{|c|c|c|c|c|c|c|c|c|c|c|c|c|c|c|c|c|c|c|c|c|c|}
\hline & \multirow{2}{*}{\multicolumn{7}{|c|}{ Groundmass }} & \multicolumn{6}{|c|}{ Hydromorphic features } & \multicolumn{8}{|c|}{ Translocation features } \\
\hline \multirow{2}{*}{ [depth $\mathrm{cm}]$} & \multicolumn{4}{|c|}{ b-fabric } & & & & \multicolumn{3}{|c|}{ nodules } & \multicolumn{2}{|c|}{ hypocoatings } & \multirow[t]{2}{*}{ dif dis } & \multicolumn{4}{|c|}{ clay coatings } & \multicolumn{3}{|c|}{ infillings } & \multirow[t]{2}{*}{ cap } \\
\hline & $\mathrm{u}$ & \begin{tabular}{l|l}
$\mathrm{ms}$ & $\mathrm{pms}$ \\
\end{tabular} & SS & pss & gs & pgs & $\mathrm{s}$ & $\mathrm{t}$ & \begin{tabular}{|l|l|}
$\mathrm{n}$ & \\
\end{tabular} & $\mathrm{a}$ & on $\mathrm{gm}$ & on cc & & $\mathrm{d}$ & \begin{tabular}{|l|l|}
$\mathrm{vd}$ & $\mathrm{l}$ \\
\end{tabular} & $1 \mathrm{~s}-\mathrm{c}$ & $f$ & \begin{tabular}{l|l}
$\mathrm{c}$ & \\
$\mathrm{c}$
\end{tabular} & $\mathrm{c}-\mathrm{s}$ & $\mathrm{s}-\mathrm{c}$ & \\
\hline IV Bt-Sd & & & & & & & & & & & & & & & & & & & & & \\
\hline 114 & & & $\mathrm{x}$ & & & $\bar{x}$ & $\mathrm{x}$ & & $\mathrm{x}$ & $\mathrm{x}$ & $\mathrm{x}$ & $\mathrm{x}$ & $\mathrm{x}$ & $\mathrm{x}$ & & $\mathrm{x}$ & & $\mathrm{x}$ & & & \\
\hline 118 & & & $x$ & & & $\bar{x}$ & $x$ & & $x$ & $\bar{x}$ & & $\bar{x}$ & $\bar{x}$ & $\mathrm{x}$ & & $x$ & & & $\mathrm{x}$ & $\mathrm{x}$ & \\
\hline 141 & & & $x$ & & & $\bar{x}$ & $x$ & & $x$ & $x$ & $\mathrm{x}$ & $\bar{x}$ & $x$ & $\mathrm{x}$ & & & & $\mathrm{x}$ & $\mathrm{x}$ & $x$ & \\
\hline & $\begin{array}{l}\text { part } \\
\text { mos } \\
\text { gran }\end{array}$ & $\begin{array}{l}\text { ly... } \\
\text { aic speck } \\
\text { ostriated }\end{array}$ & & $\begin{array}{l}\text { undi } \\
\text { stipp } \\
\text { striat }\end{array}$ & & $\begin{array}{l}\text { tiated } \\
\text { eckled }\end{array}$ & & $\begin{array}{l}\text { typ } \\
\text { nuc } \\
\text { agg }\end{array}$ & $\begin{array}{l}\text { ical } \\
\text { leic } \\
\text { regate }\end{array}$ & & $\begin{array}{l}\text { on grou } \\
\text { on clay } \\
\text { diffuse }\end{array}$ & $\begin{array}{l}\text { ndmass } \\
\text { coatings } \\
\text { distribut }\end{array}$ & & & $\begin{array}{l}\text { ty } \\
\text { y dusty } \\
\text { clay }\end{array}$ & $\begin{array}{l}\lim 1 \\
\text { frag }\end{array}$ & $\begin{array}{l}\text { pid } \\
\text { gments }\end{array}$ & & $\begin{array}{l}\text { clay } \\
\text { clay- } \\
\text { silt-c }\end{array}$ & $\begin{array}{l}\text {-silt } \\
\text { clay }\end{array}$ & \\
\hline
\end{tabular}


des Tongehaltes durch Lessivierung unwahrscheinlich erscheinen lässt (Tab. 1).

\subsubsection{Mikromorphologie}

Um eine eventuelle Lessivierung zu bestätigen, wurden vom IV Bt-Sd drei Dünnschliffe angefertigt (Tab. 2).

Neben den in-situ-Merkmalen für Ton wie etwa die Tonmineralneubildung (stipple speckled b-fabric) und die nach Јім (1986) durch physikalischen Stress verursachte Einregelung von Ton (striated b-fabric) gibt es zahlreiche Hinweise auf Tonverlagerung aus dem Hangenden. Dies zeigen teilweise vollständige tonreiche Verfüllungen von Poren und das Vorkommen von Toncutanen.

Die meisten tonhaltigen Verfüllungen der Leitbahnen (nur in den oberen beiden Proben) enthalten Siltkörner (silt-clay infillings), die auf einen relativ schnellen Transport des Tons schließen lassen (Taf. 1: 1,2).

Zwischen Toncutanen und Matrix (groundmass) ist anstatt eines scharfen oft nur ein allmählicher Übergang festzustellen (Taf. 1: 3, 4).

Die schlechte Orientierung bzw. Zerrüttung des verlagerten Tons zeigt sich an der feinmosaikartigen bzw. undulösen Auslöschung mancher Toncutane und Verfüllungen (Taf. 1: 4).

Deutlich ausgebildet sind verschiedene hydromorphe Merkmale. Fe-Oxid-Überzüge auf Toncutanen (hypocoatings on cc) weisen auf hydromorphen Einfluß nach einer Tonverlagerungsphase hin. Neben den verschiedenen konkretionären Ausbildungen (nodules) sind Fe-Oxide diffus (diffuse distribution) verteilt, mit einer deutlichen Abreicherung an Leitbahnen und gleichzeitiger Anreicherung in der Matrix (Taf. 1: 1).

\subsubsection{Genetische Schlussfolgerungen}

Zwar ist der Hauptanteil der Tongehaltserhöhung zwischen III Sw und IV Bt-Sd sedimentär bedingt, jedoch zeigen die Dünnschliffuntersuchungen anhand von Toncutanen und tonhaltigen Porenverfüllungen einen deutlichen Anteil eingewanderten Tons. Ob dieser Ton aus einer der hangenden Schichten bzw. aus Schicht III verlagert wurde, kann nicht mit Sicherheit gesagt werden.

Im Gegensatz zu der sonst typischen scharfen Abgrenzung der Toncutane zur Matrix besitzen zahlreiche Toncutane einen diffusen fließenden Übergang (Abb. 2: 3, 4), der nach SMOLIKOVA (1968) und Bronger (1976) ein Alterungsmerkmal im Sinne der Vererdung sein kann. Für LAVEs (1972) ist ein diffuser Übergang Toncutane-Matrix dagegen ein Kennzeichen für Tonverlagerung inner- halb des Bt-Horizontes bzw. für eine in-situ-Entstehung der Toncutane.

Die unter gekreuzten Polarisatoren zu beobachtende undulöse Auslöschung der Toncutane und der Porenverfüllungen ist durch eine uneinheitliche Reorientierung des Tons nach dessen Ablagerung verursacht (Taf. 1: 4). Dieses Merkmal kann ebenfalls nach der Tonverlagerung durch Quellungs- und Schrumpfungsprozesse verursacht worden sein (AltemüLleR \& BaIlly 1976).

Die Hydromorphiemerkmale scheinen alle nach oder während der Bildung der Tonverlagerungs-
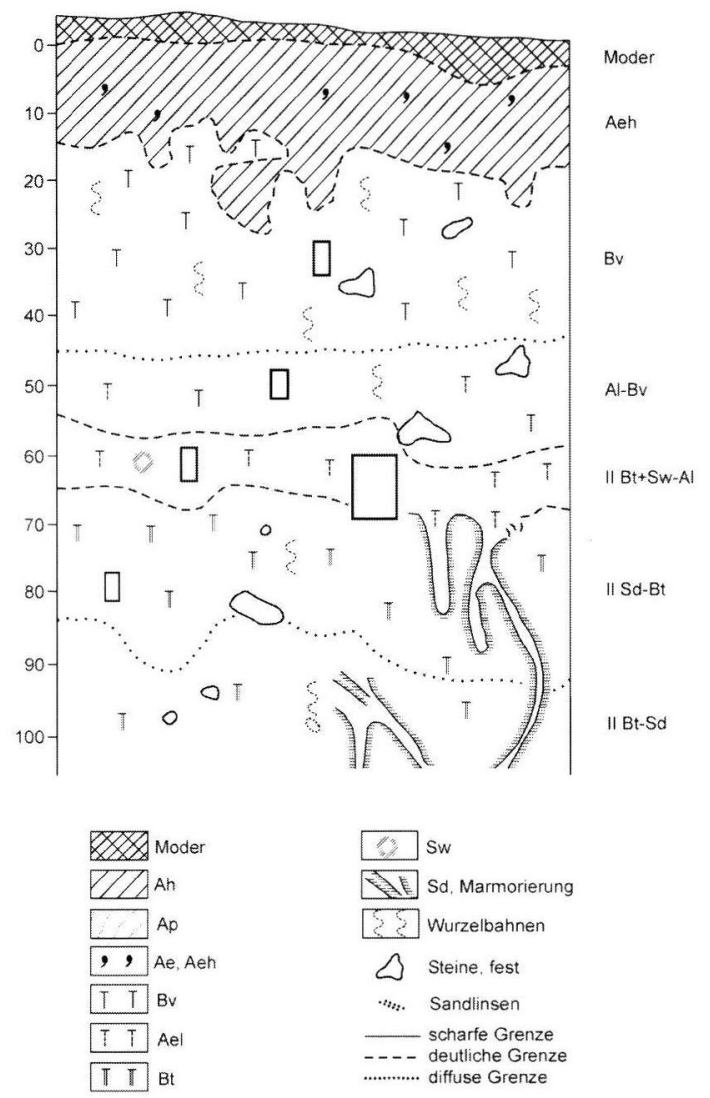

Abb. 2: Profil 2 mit Mikromorphologie-Probenahmestellen (Rechtecke). Legende nach BILLWITZ (2000).

Fig. 2: Soil profile 2; black rectangle = micromorphology sample. Legend after BiLLwiTz (2000).

merkmale entstanden zu sein, da in allen Schliffen Fe-Mn-Überzüge auf Toncutanen vorkommen (Tab. 2) und Toncutane in Leitbahnen oft „Bleichungsmerkmale" (Fe-Abreicherung) aufweisen. Eine sichere Aussage zum Alter der Toncutane 
Tab. 3: Analysedaten Profil 2.

Tab. 3: Grain size distribution and selected soil chemical data of soil profile 2.

\begin{tabular}{|c|c|c|c|c|c|c|c|c|c|c|c|c|c|c|c|}
\hline \multicolumn{16}{|c|}{ Profil 2: Mielkendorfer Gehölz 1, Wald } \\
\hline \multirow{3}{*}{ Horizont } & \multirow{3}{*}{$\begin{array}{c}\text { Tiefe } \\
{[\mathrm{cm}]}\end{array}$} & \multicolumn{12}{|c|}{ Korngrößenverteilung } & \multicolumn{2}{|c|}{$\begin{array}{c}\text { Bodenchem. } \\
\text { Kenndaten }\end{array}$} \\
\hline & & P.-tiefe & $\mathrm{T}$ & $\mathrm{fU}$ & $\mathrm{mU}$ & $\mathrm{gU}$ & fS & $\mathrm{mS}$ & $\mathrm{gS}$ & $\mathrm{S}$ & $\mathrm{U}$ & $\mathrm{T}$ & \multirow[t]{2}{*}{ B.-art } & $\mathrm{pH}$ & $\mathrm{CaCO}_{3}$ \\
\hline & & {$[\mathrm{cm}]$} & \multicolumn{10}{|c|}{$[\%]$} & & {$\left[\mathrm{CaCl}_{2}\right]$} & {$[\%]$} \\
\hline Aeh & -12 & $2-7$ & 11,8 & 3,1 & 10,0 & 14,9 & 28,0 & 24,9 & 7,2 & 60 & 28 & 12 & $\mathrm{~S} 13$ & 3,1 & - \\
\hline \multirow[t]{2}{*}{$\mathrm{Bv}$} & -47 & $15-29$ & 11,1 & 3,8 & 9,7 & 15,9 & 30,3 & 22,8 & 6,4 & 60 & 29 & 11 & $\mathrm{~S} 13$ & 3,4 & - \\
\hline & & $35-40$ & 11,1 & 3,0 & 9,9 & 15,6 & 29,1 & 22,7 & 8,6 & 60 & 29 & 11 & $\mathrm{Sl} 3$ & 4,0 & - \\
\hline $\mathrm{Al}-\mathrm{Bv}$ & -55 & $48-54$ & 9,8 & 3,6 & 9,6 & 14,8 & 28,2 & 24,5 & 9,6 & 62 & 28 & 10 & $\mathrm{SI} 3$ & 4,2 & - \\
\hline $\mathrm{Bt}+\mathrm{Sw}-\mathrm{Al}$ & -66 & $54-66$ & 7,1 & 3,9 & 8,5 & 15,6 & 31,5 & 26,1 & 7,3 & 65 & 28 & 7 & Su3 & 4,1 & - \\
\hline II Sd-Bt & -85 & $70-80$ & 19,2 & 6,0 & 8,2 & 15,6 & 29,4 & 15,6 & 6,0 & 51 & 30 & 19 & Ls4 & n.b. & n.b. \\
\hline Bt-Sd & -116 & 100-105 & 18,3 & 5,4 & 9,6 & 13,6 & 29,8 & 18,9 & 4,4 & 53 & 29 & 18 & Ls4 & 4,6 & - \\
\hline $\mathrm{Sd}$ & -147 & $130-135$ & 18,2 & 5,6 & 8,9 & 12,4 & 28,9 & 20,2 & 5,7 & 55 & 27 & 18 & Ls4 & 5,5 & - \\
\hline \multirow[t]{2}{*}{$\mathrm{Sd}-\mathrm{lCV}$} & $-180+$ & $150-155$ & 18,3 & 6,8 & 4,4 & 20,1 & 26,0 & 17,4 & 7,1 & 51 & 31 & 18 & Ls3 & 6,2 & - \\
\hline & & 180-190 & 14,2 & 2,8 & 9,5 & 14,4 & 28,6 & 24,2 & 6,4 & 59 & 27 & 14 & S14 & 7,4 & - \\
\hline
\end{tabular}

lässt sich erst treffen, wenn der Entstehungszeitraum sowohl der Toncutane in Schicht IV, als auch die Schichten I-III selbst beispielsweise physikalisch datiert werden könnten. Insgesamt kann davon ausgegangen werden, dass in dem untersuchten Horizont Ton und Schluff aus dem Hangenden eingewaschen wurde und damit eine Parabraunerde-Pseudogley-Braunerde vorliegt. Toncutane ohne hohen Siltanteil (Taf. 1: 3) werden mit LAVES \& THIERE (1970) hauptsächlich auf Lessivierung innerhalb des Bt-Sd-Horizontes zurück- geführt, die bei einem $\mathrm{pH}$-Wert von 6 auch rezent noch stattfinden kann (Tab. 1).

\subsection{Profil 2 (Mielkendorf 1)}

Profil 2 ist ein typisches Beispiel für eine pseudovergleyte Ausprägung der Braunerden über Lessivés bzw. Lessivé-Braunerden aus Decksand über Geschiebelehm (Abb. 2). Charakteristisch ist die Mächtigkeit von etwa $5 \mathrm{dm}$ der im Decksand ausgebildeten Braunerde. Die in den Sd-Bt-Horizont häufig bis in eine Tiefe von $160 \mathrm{~cm}$ reichenden,

Tab. 4: Ausgewählte mikromorphologische Merkmale von Profil 2. Klassifizierung ohne Quantifizierung nach Präsenz (Kreuz) oder Absenz (kein Kreuz). Zahlen in Klammern: mittlere Probenahmetiefe.

Tab. 4: Selected main micromorphological characteristics. The micromorphological property is shown by presence (cross) or absence (no cross). Numbers in brackets: average depth of sampling.

\begin{tabular}{|c|c|c|c|c|c|c|c|c|c|c|c|c|c|c|c|c|c|c|c|c|c|c|c|}
\hline \multirow{3}{*}{$\begin{array}{c}\text { Horizon } \\
\text { depth } \mathrm{cm}]\end{array}$} & \multicolumn{8}{|c|}{ Groundmass } & \multicolumn{6}{|c|}{ Hydromorphic features } & \multicolumn{9}{|c|}{ Translocation features } \\
\hline & \multicolumn{8}{|c|}{ b-fabric } & \multicolumn{3}{|c|}{ nodules } & \multicolumn{2}{|c|}{ hypocoatings } & \multirow[t]{2}{*}{ dif dis } & \multicolumn{5}{|c|}{ clay coatings } & \multicolumn{3}{|c|}{ infillings } & \multirow[t]{2}{*}{ cap } \\
\hline & $\mathrm{u}$ & $\mathrm{ms}$ & $\mathrm{pms}$ & SS & pss & gs & pgs & $\mathrm{s}$ & $\mathrm{t}$ & $\mathrm{n}$ & $\mathrm{a}$ & on $\mathrm{gm}$ & on cc & & $\mathrm{d}$ & $\mathrm{vd}$ & 1 & $\mathrm{~s}-\mathrm{c}$ & $\mathrm{f}$ & $\mathrm{c}$ & c-s & $\mathrm{s}-\mathrm{c}$ & \\
\hline & & & & & & & & & & & & & & & & & & & & & & & \\
\hline $\mathrm{Bv}(33)$ & $\bar{x}$ & & $x$ & & & & $\mathrm{x}$ & & & $x$ & & & & & & & & & & & & & \\
\hline $\mathrm{Al}-\mathrm{Bv}(52)$ & $\mathrm{x}$ & & & & $\mathrm{x}$ & & $x$ & & $x$ & & & & & & & & & & $\mathrm{x}$ & & & & \\
\hline $\mathrm{Bt}+\mathrm{Sw}-\mathrm{Al}(63)$ & $\bar{x}$ & & $\mathrm{x}$ & & $\bar{x}$ & & $\bar{x}$ & & & $x$ & & $\mathrm{x}$ & & & $\mathrm{x}$ & $\mathrm{x}$ & $\mathrm{x}$ & $\mathrm{x}$ & $x$ & & & & $x$ \\
\hline$(64-74)$ & $\mathrm{x}$ & & $\bar{x}$ & & & & $\bar{x}$ & & & $\bar{x}$ & $x$ & & $\mathrm{x}$ & $\mathrm{x}$ & $\bar{x}$ & $\mathrm{x}$ & $x$ & & $\mathrm{x}$ & & & $\mathrm{x}$ & $\bar{x}$ \\
\hline \multirow[t]{2}{*}{ Sd-Bt (81) } & & $\mathrm{x}$ & & & & $x$ & & & $\mathrm{x}$ & $x$ & $\mathrm{x}$ & $\mathrm{x}$ & $x$ & $\mathrm{x}$ & $\mathrm{x}$ & & $\mathrm{x}$ & $\mathrm{x}$ & & & & $x$ & \\
\hline & \multicolumn{4}{|c|}{$\begin{array}{l}\text { partly... } \\
\text { mosaic speckled } \\
\text { granostriated }\end{array}$} & \multicolumn{4}{|c|}{$\begin{array}{l}\text { undifferentiated } \\
\text { stipple speckled } \\
\text { striated }\end{array}$} & \multicolumn{3}{|c|}{$\begin{array}{l}\text { typical } \\
\text { nucleic } \\
\text { aggregate }\end{array}$} & \multicolumn{3}{|c|}{$\begin{array}{l}\text { on groundmass } \\
\text { on clay coatings }\end{array}$} & \multicolumn{3}{|c|}{$\begin{array}{l}\text { dusty } \\
\text { very dusty } \\
\text { silt-clay }\end{array}$} & \multicolumn{3}{|c|}{$\begin{array}{l}\text { limpid } \\
\text { fragments }\end{array}$} & \multicolumn{3}{|c|}{$\begin{array}{l}\text { clay } \\
\text { clay-silt } \\
\text { silt-clay }\end{array}$} \\
\hline
\end{tabular}


mit gebleichtem Material verfüllten Keilformen, werden in Verbindung mit der Redoximorphose oft als durch Quellungs- und Schrumpfungsprozesse oder Durchwurzelung verursachte Sekundärphänomene erklärt (BlumE 1968, LAATSCH 1957: 252).

\subsubsection{Analysedaten}

Die im Vergleich zum liegenden Sw-Al-Horizont um etwa 3-4\% erhöhten Tongehalte im Bv-Horizont sind für diese Profile charakteristisch (Tab. 3). Im Decksand ist keine Anreicherung im Schluffgehalt festzustellen. Die pH-Werte verhalten sich profiltypisch und zeigen an, dass unter Wald i. d. R. rezent keine Tonverlagerung anzunehmen ist (im Gegensatz zu Profilen unter Akker, vgl. Profil 3).

\subsubsection{Mikromorphologie}

Es lassen sich vier zeitlich getrennte Tonverlagerungsmerkmale feststellen (Tab. 4).

Jüngstes Verlagerungsmerkmal sind sehr staubige (vd) grau-schwarz erscheinende Toncutane, manchmal mit hohem Siltanteil, die v.a. an der Unterseite der Poren und bei mehrphasigen Cutanen immer am nächsten zum Poreninneren liegen (Taf. 2: 1). Daneben kommen schwach staubig erscheinende braune und reine gelbbraune, meist gut orientierte Toncutane (d, li) vor (Taf. 2: 2, 3). Mit den neben ungestörten Toncutanen liegenden Toncutanfragmenten (schwach staubig, rein, mikrolaminiert, gebogen) lässt sich eindeutig eine ältere Tonverlagerungsphase nachweisen. (Taf. 2: 1). Die im Al-Bv-Horizont vorkommenden Toncutanfragmente sind wahrscheinlich durchweg bioturbat fragmentiert worden. Ab einer Tiefe von etwa $80 \mathrm{~cm}$ kommen keine Toncutanfragmente mehr vor (Tab. 4).

Viertes Tonverlagerungsmerkmal sind vor allem in (ehemaligen) Leitbahnen vorkommende Verfüllungen mit silthaltigem, meist undulös auslöschendem Ton (Taf. 2: 4, 5). In Taf. 2 (5) ist eine Mehrphasigkeit der Tonverfüllung festzustellen, die sich anhand von Zonen unterschiedlich gut orientierten Tons festmachen lässt. Die roten Pfeile zeigen auf Bereiche mit deutlichen Reorientierungsmerkmalen im Ton (vgl. auch Taf. 2: 2), während weiter zum Poreninneren abgelagerter Ton besser orientiert ist. Ebenso ist eine gute Orientierung des Tons in nebenliegenden Toncutanen festzustellen.

Auf den Al- und die oberen Zentimeter des BtHorizontes beschränkt, kommen an der Oberseite von Sand- und Feinkieskörnern, nie an deren Unterseiten, bis zu mehrere $100 \mu \mathrm{m}$ mächtige schwarzgraue Feinsubstanz-Kappen (cappings) aus Ton und Silt vor (Taf. 2: 6). Selten sind darin Toncutanbruchstücke enthalten. Unter und neben diesen grobkörnigen Komponenten mit Silt-TonKappen sind häufig mit Feinsubstanz teilweise verfüllte, linsenförmige Hohlräume zu beobachten (Taf. 2: 7). In dem Verfüllungsmaterial sind Toncutanfragmente enthalten, die auf Tonverlagerung vor Entstehung und Verfüllung dieser Hohlräume hinweisen (Taf. 2: 8).

Vereinzelt lässt sich eine bänderförmige Anordnung von Grobschluff- und größeren Körnern in Verbindung mit schwach ausgeprägtem plattigen Gefüge feststellen.

Redoximorphe Merkmale in Form von diffuser FeUmverteilung (Abreicherung in der Matrix um Leitbahnen und gleichzeitiger Anreicherung in der Matrix), Fe-Mn-Konkretionsbildung und Fe-MnÜberzügen auf Toncutanen und Porenrändern sind im durch Tonverlagerung beeinflussten Profilabschnitt häufig vorhanden und besonders gut ausgebildet (Tab. 4 und Taf. 2: 4). Vereinzelt sind zwar auch oxidische Merkmale im Bv-Horizont zu finden, jedoch nur schwach ausgeprägt und dort wahrscheinlich biogen verursacht.

\subsubsection{Genetische Schlussfolgerungen}

Die jüngste, wohl auf menschlichen Einfluß zurückzuführende Ton- und Feinsubstanzverlagerung wird durch sehr staubig ausgebildete, vor allem an Porenunterseiten vorkommende Toncutane repräsentiert (Taf. 2: 1, schwarzer Pfeil, innere Lamine), die nach MACPHAIL (1986) auf Entwaldungsphasen zurückgeführt werden können. Bei schlechter Sortierung des Tonhäutchens und erkennbarem Holzkohleanteil kann dies mit einem durch Ackerbau verursachten Gefügezusammenbruch des Oberbodens zusammenhängen (JONGERIUS 1970). Die dadurch nachfolgende Feinsubstanz- bzw. Tonverlagerung wirkt sich jedoch nicht profildifferenzierend aus.

Jüngere Tonverlagerungsmerkmale sind mikrolaminierte, sehr gut bis gut orientierte Toncutane von unterschiedlich staubiger Ausprägung (Taf. 2: 2, 3), die auch im Sw-Al-Horizont zu finden sind. Bei einem Vorkommen dieser Toncutane im Al-Horizont indizieren sie eine Tonverlagerung aus dem Hangenden nach Ausbildung des Al-Horizontes. Entsprechende ausgeprägte Toncutanfragmente sind bioturbat fragmentiert worden und kommen z.B. in Bt-Schmitzen (vgl. Profil 3) nicht vor. Eine Entstehung der beschriebenen Ton-Reorientierungsmerkmale durch Wurzeln ist denkbar. Jedoch ist trotz des sauren Milieus im wurzelnahen 
Bereich anzunehmen, dass durch den Wurzeldruck fragmentierte Toncutanbruchstücke in der Pore oder porenrandnahen Matrix, sowie durch physikalische Beanspruchung entstandene Stresseigenschaften im Ton zu finden wären (vgl. Jім 1986). Weder eine Einregelung lotrecht zur angenommenen Druckrichtung noch auf Druck hinweisende Scherflächen sind im Ton zu erkennen. Als reliktische Tonverlagerungsmerkmale werden deshalb betrachtet: undulös auslöschende, v.a. in Leitbahnen vorkommende Silt-Ton-Verfüllungen mit hohem Anteil an undulös auslöschendem Ton, inaktivem Ton nach Bronger (1976) und rein bis schwach staubig erscheinende undulös auslöschende mikrolaminierte Toncutane (vgl. Kap. 4.3.3 und Diskussion).

Toncutanfragmente neben ungestörten Toncutanen und in durch Eislinsenbildung entstandenen Hohlräumen sind ebenfalls reliktisch. Diese Fragmente durchsetzen zu großen Teilen den Sw-AlHorizont und die oberen 1-2 dm des Bt-Horizontes.

Hinweise auf periglaziale Beeinflussung des SdBt-Horizontes geben die auf grobkörnigen Mineralkomponenten und Gesteinfragmenten sitzenden Silt-Ton-Kappen (Taf. 2: 6; HuJzer 1993) und meist horizontal orientierte linsenförmige Hohlräume neben und unter grobkörnigen Mineralkomponenten. Die in Tafel $2(7,8)$ vorgestellten Hohlräume lassen sich mit VANVLIET-LANOË

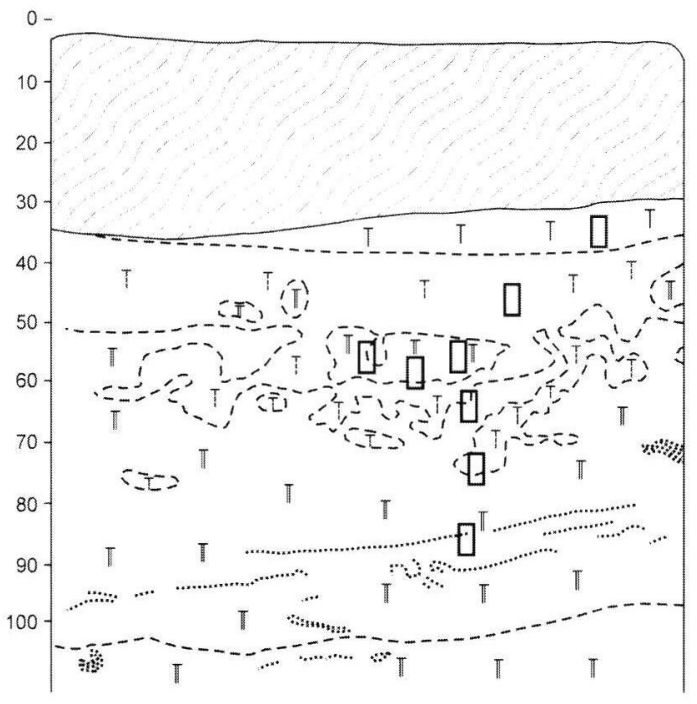

Abb. 3: Profil 3 mit Mikromorphologie-Probenahmestellen (Rechtecke); Legende siehe Abb. 2.

Fig. 3: Soil profile 3; black rectangle $=$ micromorphology sample; for legend cf. Fig. 2.
(1985: 126) zwanglos durch Eislinsenbildung erklären, durch die schon vorhandene Toncutane fragmentiert wurden. Die teilweise Verfüllung dieser Hohlräume mit Feinmaterial und Toncutanfragmenten steht dann im Zusammenhang mit dem Austauen des Permafrostes bzw. Segregationseises.

Würden die beschriebenen Merkmale durch rezenten Frosteinfluß entstehen, wäre anzunehmen, dass Toncutanfragmente in Verbindung mit frostinduzierten Mikro-Gefügemerkmalen häufiger vorkommen und auch in verschiedenen Tiefen $z u$ finden sind. Dennoch ließen sich frostgeprägte Mikro-Gefügemerkmale bisher nur in einer Tiefe von etwa 60-75 cm feststellen (KÜHN \& KÖSEL 2000, KüHN \& SCHRÖDER i. Druck, KüHN i. Druck). Es ist davon auszugehen, dass diese Merkmale reliktisch sind, da rezenter und wohl auch holozäner Frosteinfluß unter Wald aufgrund der in dieser Tiefe geringen Häufigkeit des Tau-Frostwechsels kaum gefügewirksam war. Auch ließen sich die gleichzeitig vorkommenden ungestörten Toncutane dann kaum erklären (vgl. Tab. 4).

An horizontalen Schnitten vergleichbarer Profile zeigte sich an der Obergrenze der Sd-Bt-Horizonte oft ein Polygonmuster mit einem Durchmesser der Polygone von 3-5 dm. Diese mit gebleichtem Material gefüllten zungenförmigen Keile und Polygone besitzen große Ähnlichkeit mit den von VAN Vliet \& Langohr (1981) beschriebenen Profilen im belgischen Löß, mit den Eigenschaften eines fragic horizon nach WRB (ISSS-ISRIC-FAO 1998). Aufgrund des gröberen und damit nicht so frostsuszeptiblen Substrates sind die Geländebefunde und mikromorphologischen Gefügemerkmale schwächer ausgeprägt, als von VAN VLIET \& LANGOHR (1981, 1983) im Löß gezeigt. Deshalb ist eine Polygonbildung allein durch feuchtebdingte Quellungs- und Schrumpfungswechsel an den von uns untersuchten Standorten nicht auszuschließen (Blume 1968).

Oxidische Überzüge auf Toncutanen datieren eine hydromorphe Beeinflussung auf den Zeitraum nach der Tonverlagerung (z.B. Taf. 2: 4, EisenoxidÜberzug auf Toncutane).

\subsection{Profil 3 (Me 10 I)}

Von inzwischen 13 mikromorphologisch untersuchten Lessivés in Mecklenburg-Vorpommern soll hier aus dem Darguner Raum eine „LessivéBraunerde" aus Decksand über Fließerde aus Geschiebemergel vorgestellt werden (Abb. 3).

In Profil 3 sind deutliche Fließstrukturen festzustellen, die durch pedogene Prozesse offensicht- 
Tab. 5: Analysedaten von einem vergleichbaren Profil aus der Nachbarschaft von Profil 3.

Tab. 5: Grain size distribution and selected soil chemical data of a soil profile in the neighbourhood of soil profile 3.

\begin{tabular}{|c|c|c|c|c|c|c|c|c|c|c|c|c|c|c|c|c|c|c|}
\hline \multicolumn{19}{|c|}{ Profil 3: Me 10 (I), Acker } \\
\hline & & \multicolumn{11}{|c|}{ Korngrößenverteilung } & \multicolumn{6}{|c|}{ Bodenchem. Kenndaten } \\
\hline \multirow[t]{2}{*}{ Horizont } & Tiefe & $\mathrm{T}$ & $\mathrm{fu}$ & $\mathrm{mU}$ & $\mathrm{gU}$ & $\mathrm{fS}$ & $\mathrm{mS}$ & $\mathrm{gS}$ & $S$ & $\mathrm{U}$ & $\mathrm{T}$ & B.-art & $\mathrm{pH}$ & KAK & $\mathrm{Fe}_{\mathrm{o}}$ & $\mathrm{Fe}_{\mathrm{d}}$ & $\mathrm{Fe}_{\mathrm{o}} / \mathrm{Fe}_{\mathrm{d}}$ & $\mathrm{Fe}_{\mathrm{d}} / \mathrm{Fe}_{\mathrm{t}}$ \\
\hline & {$[\mathrm{cm}]$} & \multicolumn{10}{|c|}{$[\%]$} & & {$\left[\mathrm{CaCl}_{2}\right]$} & [mval/100g] & [Gew.\%] & [Gew.\%] & & \\
\hline $\mathrm{Ah}$ & -33 & 5,4 & 2,2 & 6,8 & 24,6 & 39,5 & 18,5 & 3,0 & 61 & 34 & 5 & Su3 & 4,7 & 2,7 & 0,13 & 0,38 & 0,34 & 0,60 \\
\hline $\mathrm{Bv}$ & -55 & 6,0 & 2,6 & 6,9 & 22,0 & 38,3 & 21,0 & 3,4 & 63 & 32 & 6 & Su3 & 5,2 & 1,4 & 0,09 & 0,29 & 0,31 & 0,48 \\
\hline II Ael & -70 & 4,2 & 3,1 & 6,6 & 16,0 & 40,9 & 25,0 & 4,3 & 70 & & 4 & Su3 & 5,6 & 1,0 & 0,04 & 0,22 & 0,18 & 0,39 \\
\hline $\mathrm{Bt}$ & $>70$ & 12,1 & 3,1 & 5,9 & 11,5 & 41,0 & 23,0 & 3,5 & 68 & 21 & 12 & S14 & 5,4 & 2,7 & 0,07 & 0,47 & 0,15 & 0,44 \\
\hline
\end{tabular}

lich nicht homogenisiert werden konnten. Es ist kein keilförmiges Eingreifen des Al-Horizontes in den Bt-Horizont wie bei Profil 2 festzustellen, sondern ein nach oben in „Bt-Schmitzen“ aufgelöster Bt-Horizont. Die im Al+Bt-Horizont „schwimmenden Bt-Schmitzen“ verschiedener Größe sind ein häufig zu beobachtendes Merkmal, das hier besonders eindrucksvoll ausgebildet ist. Bt-Bänder zwischen den Bt-Schmitzen, wie sie von Roeschmann (1968) beschrieben wurden, lieBen sich hier nicht feststellen.

\subsubsection{Analysedaten}

Neben den von Schröder \& Schneider (1996) und SCHRÖDER et al. (1997) vorgestellten Daten zu Lessivé-Braunerden im Darguner Raum sei auf einige Charakteristika noch einmal hingewiesen (vgl. Tab. 5). Die Daten von Profil 3 stammen von einem einige Jahre vorher beprobten, wenige Meter entfernten Profil, in dem der Ael+Bt-Horizont undeutlich ausgeprägt war.

Bei etwa einem Drittel der untersuchten Profile besitzt der Decksand eine deutliche Schluffanreicherung einhergehend mit einer Zunahme des gUGehaltes am Gesamtschluff. Der Bv-Horizont ist im Vergleich zum liegenden Ael-Horizont durch eine Erhöhung des Tongehaltes gekennzeichnet, die bei allen von uns untersuchten Profilen in Mecklenburg-Vorpommern zwischen 2-4\% beträgt. Dies ist auch bei Profil 2 aus Schleswig-Holstein festzustellen. Insgesamt sind die Profile sehr sandig. Wie zu erwarten, sind die Werte für den Aktivitätsgrad im $\mathrm{Ah}$ und $\mathrm{Bv}$ höher als im Liegenden, da der Alterungsprozess des pedogenen Ei-

Tab. 6: Ausgewählte mikromorphologische Merkmale von Profil 3. Klassifizierung ohne Quantifizierung nach Präsenz (Kreuz) oder Absenz (kein Kreuz); st = nur in Bt-Schmitzen vorkommend. Zahlen in Klammern: mittlere Probenahmetiefe.

Tab. 6: Selected main micromorphological characteristics. The micromorphological property is shown by presence (cross) or absence (no cross); st = in Bt-streaks only. Numbers in brackets: average depth of sampling.

\begin{tabular}{|c|c|c|c|c|c|c|c|c|c|c|c|c|c|c|c|c|c|c|c|c|c|c|c|c|}
\hline \multirow{3}{*}{\begin{tabular}{|c|} 
Horizon \\
{$[$ depth $\mathrm{cm}]$} \\
\end{tabular}} & \multicolumn{8}{|c|}{ Groundmass } & \multicolumn{6}{|c|}{ Hydromorphic features } & \multicolumn{10}{|c|}{ Translocation features } \\
\hline & \multicolumn{8}{|c|}{ b-fabric } & \multicolumn{3}{|c|}{ nodules } & \multicolumn{2}{|c|}{ hypocoatings } & \multirow{2}{*}{ dif dis } & \multicolumn{5}{|c|}{ clay coatings } & \multicolumn{3}{|c|}{ infillings } & \multirow{2}{*}{ cap } & \multirow[t]{2}{*}{ st } \\
\hline & $\mathrm{u}$ & $\mathrm{ms}$ & pms & ss & pss & gs & pgs & $\mathrm{s}$ & $\mathrm{t}$ & $\mathrm{n}$ & $\mathrm{a}$ & on gm & on cc & & $\mathrm{d}$ & \begin{tabular}{|l|}
$\mathrm{vd}$ \\
\end{tabular} & 1 & $s-c$ & $f$ & $\mathrm{c}$ & $\mathrm{c}-\mathrm{s}$ & $\mathrm{s}-\mathrm{c}$ & & \\
\hline & & & & & & & & & & & & & & & & & & & & & & & & \\
\hline Bv (37) & $\mathrm{x}$ & & $\mathrm{x}$ & & & & & & & & $x$ & & & & & & $\mathrm{x}$ & & $\mathrm{x}$ & & & & & \\
\hline Ael (47) & $x$ & st & & & & st & & & & $x$ & $x$ & & & & $\mathrm{xst}$ & $\mathrm{x}$ & & st & $\mathrm{xst}$ & & & & $\mathrm{x}$ & $x$ \\
\hline Ael (48) & $\mathrm{x}$ & st & & & & & st & & & $x$ & & $x$ & & & $\mathrm{x}$ & $\mathrm{x}$ & & & xst & & & $\mathrm{x}$ & $\mathrm{x}$ & $x$ \\
\hline $\mathrm{Ael}+\mathrm{Bt}(56)$ & $\mathrm{x}$ & st & & & & & & & & $x$ & & $\mathrm{x}$ & & & $\mathrm{x}$ & $\mathrm{x}$ & $\mathrm{x}$ & & xst & & & $\mathrm{x}$ & $\mathrm{x}$ & $\mathrm{x}$ \\
\hline $\mathrm{Ael}+\mathrm{Bt}(57)$ & $x$ & & St & & & & st & & & $x$ & & & & & $x$ & $x$ & & & xst & & & & $x$ & $x$ \\
\hline $\mathrm{Ael}+\mathrm{Bt}(60)$ & $\mathrm{x}$ & & st & & & & st & & & $\mathrm{x}$ & & & & & & $\mathrm{x}$ & & $x$ & $x$ & & & & $\mathrm{x}$ & $\mathrm{x}$ \\
\hline $\mathrm{Bt}(67)$ & & & $x$ & & & & $x$ & & & $x$ & & & & & $\mathrm{x}$ & $\mathrm{x}$ & & $\mathrm{x}$ & $x$ & $x$ & & $\mathrm{x}$ & & \\
\hline $\mathrm{Bt}(80)$ & & & $\mathrm{x}$ & $\mathrm{x}$ & & & & & & $\mathrm{x}$ & & & $\mathrm{x}$ & & $\mathrm{x}$ & $\mathrm{x}$ & $\mathrm{x}$ & & & & & $\mathrm{x}$ & & \\
\hline $\mathrm{Bt}(90)$ & & & $\mathrm{x}$ & & & & $\mathrm{x}$ & & & $\mathrm{x}$ & $\mathrm{x}$ & & & & $\mathrm{x}$ & & $x$ & & & & $x$ & $x$ & & \\
\hline & \multirow{2}{*}{\multicolumn{3}{|c|}{$\begin{array}{l}\text { partly... } \\
\text { mosaic speckled } \\
\text { granostriated }\end{array}$}} & \multicolumn{5}{|c|}{$\begin{array}{l}\text { undifferentiated } \\
\text { stipple speckled } \\
\text { striated }\end{array}$} & \multirow{2}{*}{\multicolumn{3}{|c|}{$\begin{array}{l}\text { typical } \\
\text { nucleic } \\
\text { aggregate }\end{array}$}} & \multirow{2}{*}{\multicolumn{3}{|c|}{$\begin{array}{l}\text { on groundmass } \\
\text { on clay coatings }\end{array}$}} & \multirow{2}{*}{\multicolumn{3}{|c|}{$\begin{array}{l}\text { dusty } \\
\text { very dusty } \\
\text { silt-clay } \\
\text { on }\end{array}$}} & \multirow{2}{*}{\multicolumn{2}{|c|}{$\begin{array}{l}\text { limpid } \\
\text { fragments }\end{array}$}} & & $\begin{array}{l}\text { clay } \\
\text { silt-c }\end{array}$ & clay & \multicolumn{2}{|c|}{$\begin{array}{l}\text { clay-silt } \\
\text { capping }\end{array}$} \\
\hline & & & & & & & & & & & & & & distribut & & & & & & & & & Bt-st & \\
\hline
\end{tabular}


sens noch nicht sehr weit fortgeschritten ist. Erstaunlich, aber in allen Profilen wiederzufinden, ist der höhere Anteil an dithionitlöslichem Eisen am Gesamteisen $\left(\mathrm{Fe}_{\mathrm{d}} / \mathrm{Fe}_{\mathrm{t}}\right)$ im $\mathrm{Ah}$ - und Bv-Horizont im Vergleich zum Ael- und Bt-Horizont. Die höchsten $\mathrm{Fe}_{\mathrm{d}}$-Werte wären aufgrund der Tonverlagerung im Bt-Horizont zu erwarten gewesen. Daher kann nur eine intensivere Verwitterung nach der Tonverlagerung zu den deutlich höheren $\mathrm{Fe}_{\mathrm{d}} /$ $\mathrm{Fe}_{\mathrm{t}}$-Werten der Braunerde geführt haben.

\subsubsection{Mikromorphologie}

Die in Tabelle 6 ausgewählten Merkmale zeigen zum großen Teil die erwartete Tiefenverteilung. Die getroffene Unterteilung in Bt-Schmitzen (st) gebundene und matrixgebundene (x) Merkmale soll die unterschiedliche Ausprägung beider Bereiche hervorheben, da neben der undifferentiated b-fabric weitere b-fabrics im Ael-Horizont sonst nicht zu erklären wären.

Typisch für Ael- und Ael+Bt-Horizonte sind TonSilt-Kappen auf Sandkörnern oder Gesteinsfragmenten, deren Feinsubstanz im Durchlicht meist schwarzgrau erscheint (vgl. KEMP et al. 1998). Oft sind diese Kappen auch zweiphasig mit tonreicher Basis ausgebildet (Taf. 3: 1, 2). Die Bildung dieser tonreichen Kappen (untere Phase) wird von HuIZER (1993: 182, 200) auf vertikale Verlagerung von Feinmaterial durch das Austauen des hangenden Bodens bzw. auf die saisonale Existenz der Auftauzone zurückgeführt. Im Schliff bei $47 \mathrm{~cm}$ finden sich zahlreiche Kappen mit seitlich geneigter Orientierung an der Kornoberfläche, die teilweise auch schon makromorphologisch zu erkennen sind (Taf. 3: 3). Die von Huijzer (1993: 178) festgestellte Verknüpfung seitlich geneigter Kappen mit makro- bzw. mikroskopischen Kryoturbationsmerkmalen ließ sich hier jedoch nicht nachweisen.

Bt-Schmitzen (oft $<1 \mathrm{~cm}$ Durchmesser) sind nur im Ael- und Ael+Bt-Horizont vorhanden (Taf. 3: 3). Sie sind in sich relativ homogen, besitzen verschiedene Merkmale orientierten Tons (b-fabrics) in der Matrix. Zudem finden sich in den Bt-Schmitzen manchmal schwer zu identifizierende Toncutanbruchstücke (Taf. 3: 4, vgl. auch SCHNEIDER \& KüHN 2000).

Staubige Toncutane und konkave Toncutanbrücken zwischen Mineralen und Aggregaten im AelHorizont bezeugen Tonverlagerung aus der hangenden Braunerde. Sehr staubige grauschwarze Toncutane sind hauptsächlich an Porenunterseiten ausgebildet und kommen vom Ael- bis zum
Bt-Horizont vor. Bei zusammengesetzten Toncutanen liegen diese immer am nächsten zum Poreninneren (Taf. 3: 5, 6).

Ab etwa $65 \mathrm{~cm}$ Tiefe liegen neben sehr gut orientierten (scharfe Auslöschungsbänder) im Durchlicht gelbbraunen Toncutanen auch schlecht orientierte undulös auslöschende, im Durchlicht gelbbraune bis braune Toncutane, die ein unterschiedliches Bildungsalter nahe legen (Taf. 4: 1,2).

Verfüllungen sind v.a. für den Bt-Horizont typisch, aber auch (sehr selten) im Ael-Horizont ausgebildet. Meist löschen sie undulös aus und sind nur selten frei von Schluffkörnern. In Tafel $4(3,4)$ sind zweiphasige, sehr gut orientierte Toncutane an eine undulös auslöschende Silt-Ton-Verfüllung angelagert. In Tafel 4 (3) sind die verschiedenen Tonverlagerungsphasen klar erkennbar, während in Tafel 4 (4) die unterschiedlichen Auslöschungseigenschaften des illuviierten Tons deutlich werden.

Wie in Profil 2 beschränkt sich das Vorkommen von Toncutanfragmenten v.a. auf den Ael-Horizont und die oberen 1-2 dm des Bt-Horizontes. Hydromorphiemerkmale in Form von Fe-OxidAusfällungen in der Matrix und Überzügen auf Porenrändern und vereinzelt auf Toncutanen sind zwar vorhanden, besitzen jedoch im Gegensatz zu Profil 2 keine prägende Auswirkung.

\subsubsection{Genetische Schlußfolgerungen}

Es sind unterschiedlich alte Tonverlagerungsphasen anhand verschiedener Toncutangenerationen festzustellen.

Zur älteren Tonverlagerungsphase zählen undulös auslöschende Toncutane und Verfüllungen sowie meist gebogene gelbbraune, manchmal mikrolaminierte Toncutanfragmente. Ist die Fragmentierung der Toncutane anhand der Mikro-Gefügemerkmale auf Bioturbation zurückzuführen, kann i.d.R. keine relative chronologische Einordnung vorgenommen werden.

Zur jüngeren Tonverlagerungsphase zählen gut orientierte Toncutane mit scharfen Auslöschungsbändern und alle Toncutane im Ael- und Ael+BtHorizont, die dort meist als konkave Tonbrücken zwischen den Mineralen ausgebildet sind.

Die jüngste, anthropogen verursachte Ton- bzw. Feinsubstanzverlagerung kann anhand von grauschwarzen siltreichen Toncutanen (vd) festgestellt werden, die meist nur an den Porenunterseiten ausgebildet sind (Erklärung siehe Kap. 4.2.3).

Bei einmal abgelagerten Toncutanen unterscheidet FitzPatrick (1993: 184) fünf Stufen der Gefüge-Reorganisation, die einen in-situ-Alterungs- 

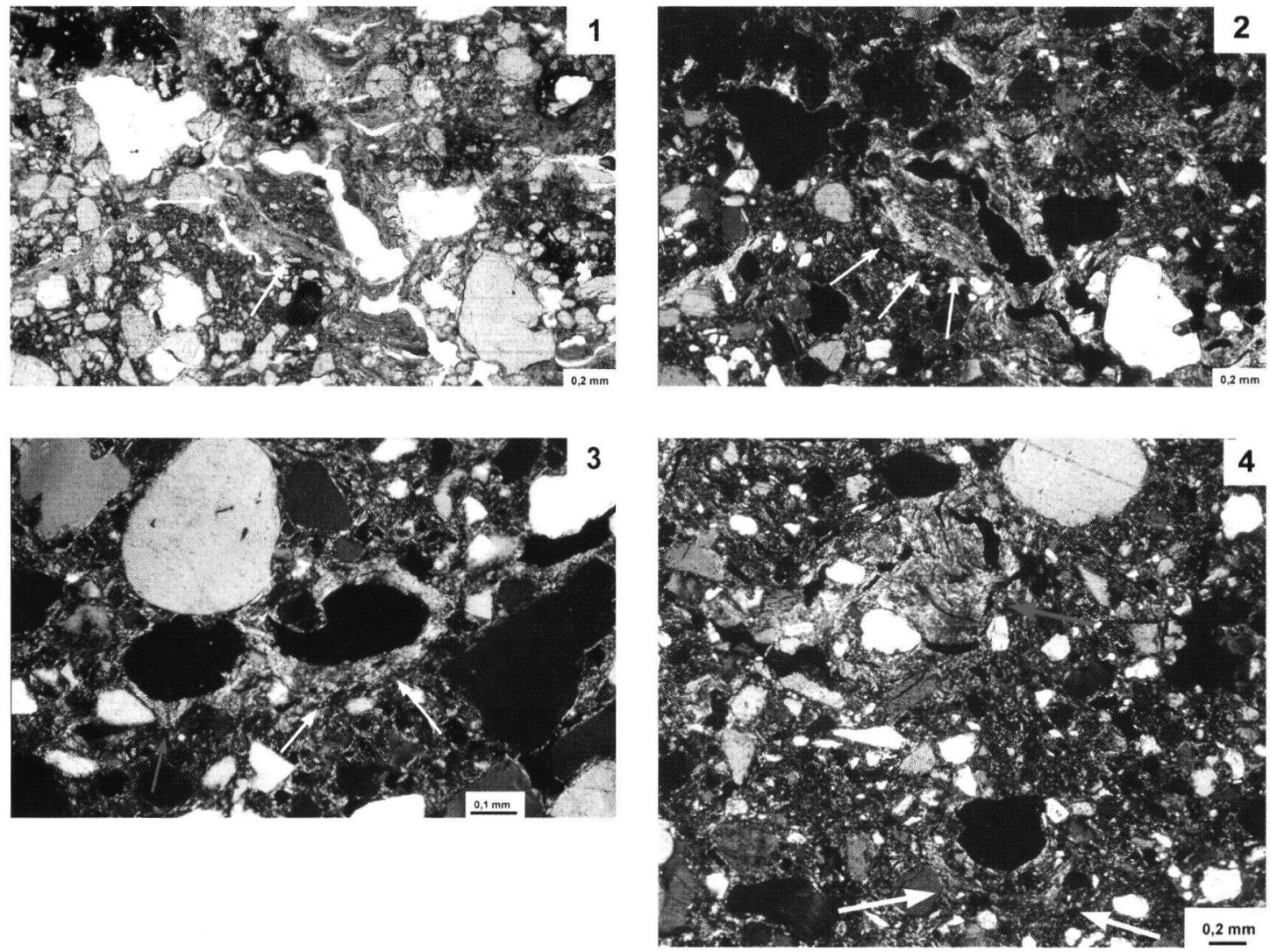

Tafel 1: Dünnschliffbilder von Profil 1. (1) $118 \mathrm{~cm}$ : Mehrphasige Schluff-Ton-Verfüllung (Pfeile) und diffuse Fe-Umverteilungsmerkmale in der Matrix (dunkle Flächen) - Durchlicht. (2) $118 \mathrm{~cm}$ : Photo (1) mit gekr. Pol. (3) $141 \mathrm{~cm}$ : schlecht orientiertes Tonhäutchen (weiße Pfeile) mit diffusem Übergang (Alterungsmerkmal: „Vererdung“) zur Matrix und sehr gut orientiertes Tonhäutchen mit scharfem Auslöschungsband (roter Pfeil) - gekr. Pol. (4) $118 \mathrm{~cm}$ : undulös auslöschende Silt-Ton-Verfüllung (roter Pfeil) mit teilweise diffusem Übergang zur Matrix und mehrphasiges Tonhäutchen mit diffusem Übergang zur Matrix (weiße Pfeile) - gekr. Pol.

Plate 1: Photomicrographs of soil profile 1. (1) $118 \mathrm{~cm}$ : Compound silt-clay-infilling (arrows) and diffuse iron-oxide-distribution in the groundmass (dark areas) - ppl. (2) $118 \mathrm{~cm}$ : Photomicrograph (1) with cpl. (3) $141 \mathrm{~cm}$ : Weakly orientated clay coating (white arrows) with diffuse transition to the groundmass (ageing effect: "Vererdung"); red arrows: well orientated clay coating with diffuse transition to the groundmass - cpl. (4) $118 \mathrm{~cm}$ : silt-clay-infilling with undulatory extinction pattern (red arrow) and compound clay coating with diffuse transition to the groundmass (white arrows) $-\mathrm{cpl}$.

Tafel 2: Dünnschliffbilder von Profil 2. (1) Zweiphasiges Toncutane (schwarzer Pfeil) und reines mikrolaminiertes gebogenes Toncutanbruchstück (roter Pfeil) - 64-74 cm, Durchlicht. (2) Zweiphasige Toncutane mit jüngerer staubiger Phase (schwarze Pfeile) - 63 cm, Durchlicht. (3) Scharfe begrenzte Auslöschungsbänder (weiße Pfeile) zeigen die sehr gute Orientierung des Tons - wie (2), gekr. Pol. (4) Silt-Ton-Verfüllung in der Mitte des Photos; diffuse Fe-Mn-Oxid-Ausscheidung (dunkle Flächen) und Fe-Oxid-Überzüge auf Toncutanen (kurzer roter Pfeil) - $81 \mathrm{~cm}$, Durchlicht. (5) Silt-Ton-Verfüllung mit deutlichen Reorientierungsmerkmalen des Tons (rote Pfeile) während andere Toncutane sehr gut orientiert sind (rechts: kurze weiße Pfeile) - wie (4), gekt. Pol. (6) Ton-Silt-Kappe auf Feinkies - 64-74 cm, Durchlicht. (7) Mit Feinsubstanz (schwarze Pfeile) teilweise verfüllter Hohlraum unterhalb des Feinkieses mit „Kappe“ von Photo (6) $-64-74 \mathrm{~cm}$, Durchlicht. (8) Unterschiedlich gut orientierte Toncutanbruchstücke (weiße Pfeile) in Hohlraumverfüllung - wie (7) mit gekr. Pol.

Plate 2: Photomictographs of soil profile 2. (1) Compound clay coating (black arrow) and limpid microlaminated bent fragment of clay coating (red arrow) $-64-74 \mathrm{~cm}$, ppl. (2) Compound clay coating with younger dusty lamina (black arrows) $-63 \mathrm{~cm}$, ppl. (3) Perfectly orientated clay coatings characterized by extinction bands (white arrows) with sharp boundaries - (2) with cpl. (4) Silt-clay-infilling in the centre of the photomicrograph; diffuse distributed Fe-Mn-oxide-precipitations (dark brownish areas) and ferrugineous hypocoatings on clay coating (small red arrow) $-81 \mathrm{~cm}$, ppl. (5) Silt-clay infilling with distinct features of reorientation of clay (red arrows) beside well orientated clay coatings (small white arrows) - photomicrograph (4) with cpl. (6) Clay-silt-capping on fine gravel grain - 64-74 cm, ppl. (7) Partially infilled void beneath fine gravel grain with capping from photomicrograph (6) - 64-74 cm, ppl. (8) photomicrograph (7) with cpl. 


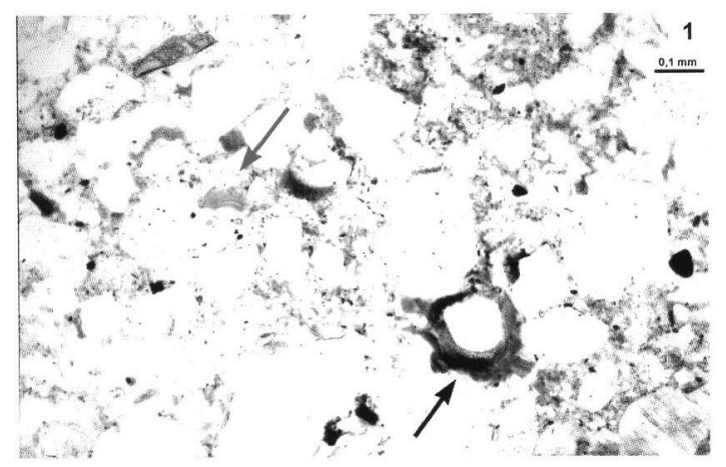

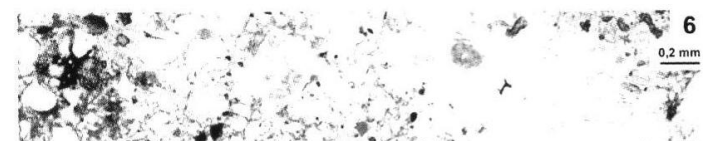
A.

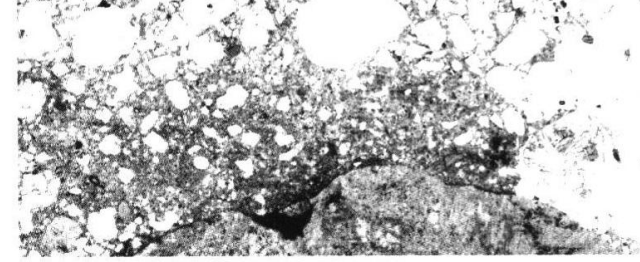
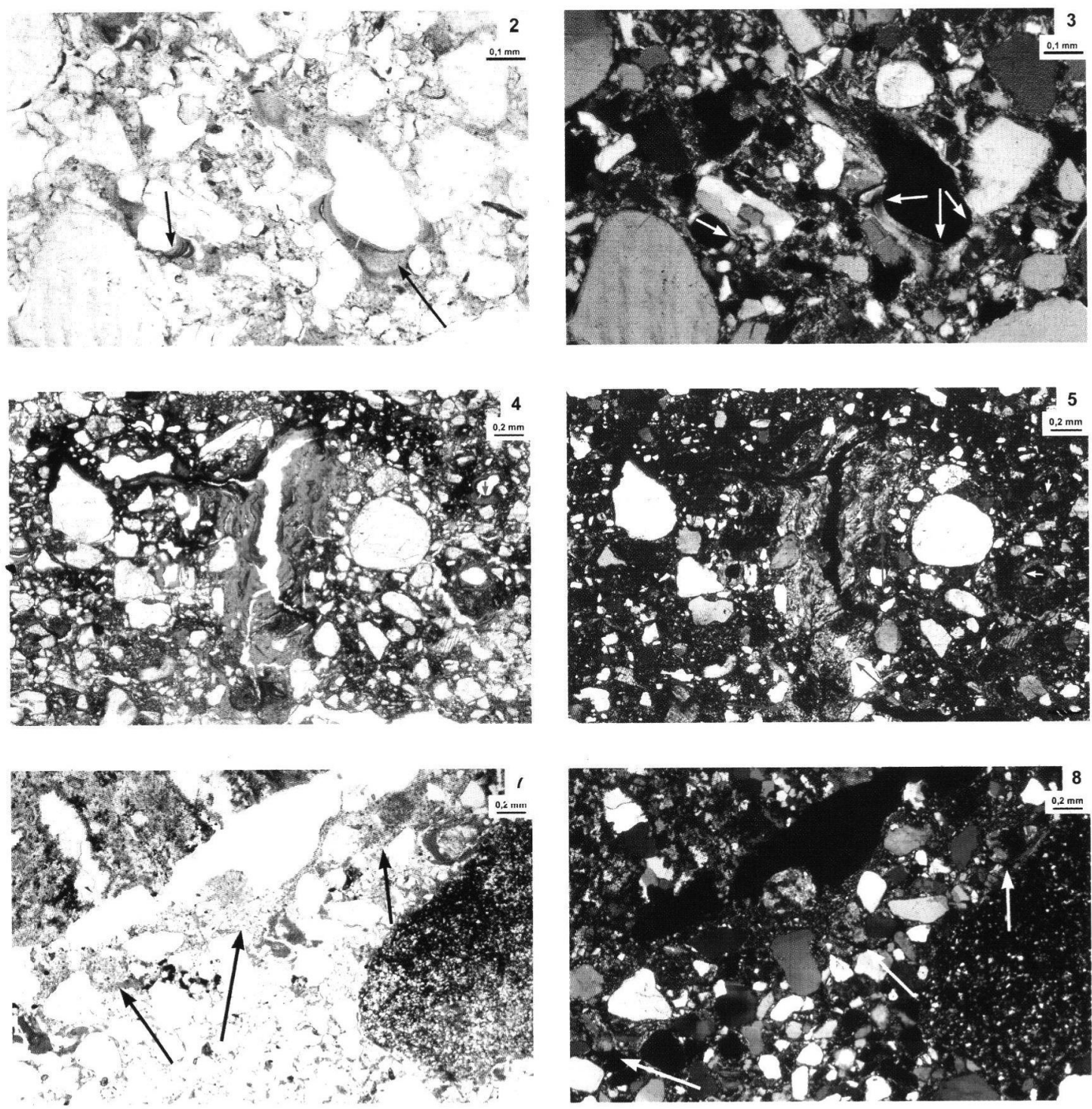
prozeß beschreiben. Entscheidend für die Einstufung ist der prozentuale Anteil an undulös auslöschenden Bereichen in der Gesamtcutane. FrTZPATRICK (1993: 182) bezeichnet dieses Auslöschungsmuster als ,granular extinction pattern", das nicht mit der in Kap. 2 angesprochenen „Granulierung “ zu verwechseln ist. Das Nebeneinander von undulös auslöschenden und sehr gut orientierten Toncutanen (Taf. 4) legt deshalb ein unterschiedliches Alter nahe, da nach Bildung der Toncutane eine Beeinflussung durch Bioturbation oder "Artefaktbildung“ bei der Dünnschliffherstellung ausgeschlossen werden kann.

\section{Zusammenfassende Diskussion}

In Profil 1 ließ sich mikromorphologisch zunächst der Nachweis der Lessivierung führen. Aufgrund der tiefen Lage des Bt-Horizontes und der oft undeutlichen Abgrenzung der Toncutane zur umgebenden Matrix, im Gegensatz zu Toncutanen in Profil 2 und 3, wird für siltarme Toncutane eine Tonverlagerung überwiegend innerhalb des BtHorizontes angenommen, die auch rezent noch stattfinden kann (LAVES \& THIERE 1970).

Mit Profil 2 und 3 wurden zwei häufig vorkommende Ausprägungen der Braunerde-Lessivés vorgestellt: Profil 2 als Beispiel für zungen- bis keilförmiges Eingreifen des Al-Horizontes in den Bt-Horizont (Typ 1) und Profil 3 als Beispiel für eine fleckenförmig aufgelöste Obergrenze des BtHorizontes (Typ 2).

Zunächst stellt sich die Frage, ob es sich bei den Merkmalen im Übergangsbereich zwischen Alund Bt-Horizonten nicht einfach um eine Tieferlegung der Bt-Horizonte im Zuge der Pedogenese handelt. Gegen eine Merkmalsausprägung durch einfache Degradation der oberen Bereiche der untersuchten Bt-Horizonte im Sinne einer Tieferlegung sprechen:

1) bei Typ 1 die Toncutanbruchstücke ohne Reaktivierungsmerkmale im Al-Horizont, sowie gut erhaltene Toncutane im Al-Horizont als Merkmal einer jungen Tonverlagerungsphase,

2) bei Typ 2 die Toncutanbruchstücke in isolierten Bt-Schmitzen, die wie in Profil 3 im Übergangsbereich zwischen $\mathrm{Al}$ - und Bt-Horizonten bzw. in $\mathrm{Al}+\mathrm{Bt}-$ Horizonten $\mathrm{zu}$ finden sind, und

3) fehlende Degradationserscheinungen von BtAggregaten im Sinne von Bullock et al. (1974), die eine Tonverarmungszone am Rand von Aggregaten mit tonhaltigem Kern beschreiben.

Weiterhin ist eine Auflösung der Bt-Horizonte durch das Mitreißen von Bodenmaterial in Wur- zeltellern durch Windwürfe denkbar (ROESCHMANN 1968). Dies ist nicht völlig auszuschließen, doch sind im Gelände weitere auf Windwurf hinweisende Merkmale, wie von LANGOHR (1993) und Russow \& HeInRICH (2001) diskutiert, in den untersuchten Profilen nicht vorzufinden. Deshalb gehen wir davon aus, dass die Bt-Schmitzen älter und nicht durch Windwurf entstanden sind.

Profilmorphologische Kennzeichen wie Fließerden (Profil 3) über dem Bt, Sandkeile und Kryoturbationsmerkmale im Bt weisen auf ein kalt-arides Klima mit Permafrost hin (Helbig 1999: 64), durch das die Grundmoränenplatten im Jüngsten Jungmoränengebiet im Spätglazial eine nicht unerhebliche periglaziale Überprägung erfuhren. Schon LAVES (1972) nahm eine periglaziale Genese der Bt-Schmitzen an. Da diese inzwischen auch in Sandkeilfüllungen beschrieben wurden (KÜHN i. Druck; bei HeLBIG 1999: 60 als till spots bezeichnet), verstärken sich im Zusammenhang mit den hier vorgestellten Befunden die Hinweise auf eine spätglaziale periglaziale Umlagerung der oberen Bereiche schon bestehender Bt-Horizonte. Diese Annahme wird zusätzlich durch in anderen Profilen makroskopisch erkennbare Bt-Schmitzen bestätigt, die in Füllungen von Sandkeilen periglaziärer Genese vorkommen (KüHN i. Druck). Unterstützt wird dies durch die makroskopischen Fließerdemerkmale im Bt-Horizont von Profil 3, sowie durch die von Helbig (1999: 61) in der Nähe von Barlin beschriebenen Sandkeile, deren Schulterbereiche auf flach geneigten Hängen durch Solifluktion hangabwärts verzogen sind.

Mikromorphologisch geben die deutlich ausgeprägten, auf Sandkörnern oder Gesteinsfragmenten liegenden Ton-Silt-Kappen mit tonreicher Basis weitere Hinweise auf periglazialen Einfluß und mit großer Wahrscheinlichkeit auch die undulös auslöschenden Toncutane und Silt-Ton-Verfüllungen im Bt-Horizont. Dass trotz makroskopischer Belege mikromorphologisch-periglaziale Merkmale nur schwach ausgebildet sind oder fehlen, kann nach Hujzer (1993) am sandigen Substrat und den geringen primären Körnungsunterschieden der Schichten liegen.

Somit wären die in die Bt-Schmitzen inkorporierten Toncutanfragmente ein Beweis für eine Tonverlagerung vor der periglazialen Beeinflussung und Umlagerung des oberen Bereiches des BtHorizontes. Einen ähnlichen genetischen Zusammenhang zwischen Ton-Silt-Kappen, Toncutanfragmenten und makroskopischen Kryoturbationsmerkmalen konnten TARNOCAI \& SMITH (1989) in Paläoböden in Yukon (Canada) feststellen. 
Von einer Lessivierungsphase vor dieser Umlagerung zeugen sowohl die in die Bt-Schmitzen inkorporierten Toncutanfragmente, als auch die in Sandkeilfüllungen mikromorphologisch nachgewiesenen Toncutanfragmente (KüHN i. Druck, KÜHN \& SCHRÖDER i. Druck).

Bei normaler Profil- und Substratausprägung sind ab etwa $80 \mathrm{~cm}$ Tiefe, außer den eindeutig bioturbat fragmentierten, keine Toncutanbruchstücke mehr festzustellen. Auch in dieser Tiefe kommen neben den gut orientierten Toncutanen zahlreiche schlecht orientierte undulös auslöschende Toncutane vor, die nach FITZPATRICK (1993) durch in-situAlterung hervorgerufen werden (siehe Kap. 4.3.3). Doch worin liegt die Ursache für diese „Alterung und welchen Zeitraum umfasst sie? Ein Zeitraum für die Entstehung der Alterungsmerkmale kann bisher nicht angegeben werden. AltemülleR \& BAILLY (1976) stellen fest, dass auch rezent noch stattfindende Quellungs- und Schrumpfungsprozesse nicht zu einer Desorientierung aller Toncutane in (Pseudogley-)Lessivés geführt haben. Wären jedoch jene Prozesse allein für eine GefügeReorganisation verantwortlich, so müssten alle Toncutane bzw. Porenverfüllungen davon betroffen sein. Denkbar ist eine spätglaziale Beeinflussung der schon in Interstadialen gebildeten Toncutane durch Gefrieren des Bodens in Stadialen. Dadurch ließe sich das Nebeneinander von sehr gut und sehr schlecht orientierten Toncutanen in kaum hydromorph beeinflussten Lessivés zwanglos erklären.

Der hohe Sandgehalt der Geschiebemergel (meist $>50 \%$ ) und Decksande (>50-60\%) im norddeutschen Jungmoränengebiet ermöglicht nach der Entkalkung aufgrund des schon vorhandenen Porenvolumens eine ,schnelle“ vertikale Verlagerung des Tons. Im Untersuchungsraum in Mecklenburg-Vorpommern besitzen die Geschiebemergel $\left(\mathrm{CaCO}_{3}\right.$-Gehalt zwischen 10-15\%) nach deren Entkalkung in der Feinerde $(<2 \mathrm{~mm})$ einen Tongehalt von etwa $15 \%$. Dadurch ist schon, ohne vorher stattgefundene Silikatverwitterung, ein Potential an verlagerbarem Ton vorhanden. Wie von STEPHAN (2000) beschrieben, kann unter diesen Voraussetzungen der im Sediment vorhandene Ton schon sofort nach der Entkalkung entlang des vorliegenden Porenraums verlagert werden. Es muß deshalb nicht erst eine Neubildung und Wanderung des Tons durch die Matrix hin zu den Grobporen angenommen werden, um zu einer Horizontdifferenzierung durch Lessivierung zu gelangen.

Zuletzt lassen auch die mindestens 3000-4000 Jah- re Eisfreiheit im Untersuchungsgebiet vor dem Holozän für spätglaziale Entcarbonatisierung mit nachfolgender Tonverlagerung einen genügend langen Zeitraum. Für das „Meiendorf/Bölling““ zeigt die Rekonstruktion der spätglazialen Paläoumweltbedingungen an der Lokalität Reinberg (Vorpommern) den Eintrag carbonatfreien Hangwassers in das Reinberg-Becken, anhand dessen DE KLERK (2001) schon im „Meiendorf/Bölling“ das Vorhandensein von im Sickerwassersaum entkalkter Böden annimmt. Die Übergänge von den kälteren zu den wärmeren spätglazialen Chronozonen haben sich überregional z.T. innerhalb weniger Jahrzehnte vollzogen (BERNER \& STREIF 2000, KaISER 1993), so dass sich dadurch die Voraussetzungen für eine weitergehende Pedogenese sehr schnell verbessern konnten. Gerade bei einem teilweisen Austauen des Permafrostes während der Interstadiale ist mit einer verstärkten vertikalen Ton- und Feinsubstanzverlagerung zu rechnen, die mit großer Wahrscheinlichkeit durch die undulös auslöschenden, gealterten Silt-Ton-Verfüllungen repräsentiert sind (Taf. 2: 5; Taf. 4: 3, 4). Anhand der vorgestellten Befunde ergibt sich folgende, wenn auch grobe chronologische Abfolge pedogenetischer Prozesse in den untersuchten Leitböden:

Spätglazial: Entkalkung - (Verbraunung) (Hydromorphierung bzw. Redoximorphose i.V.m. Permafrost) - Lessivierung: Toncutanfragmente in Bt-Schmitzen, Silt-Ton-Verfüllungen und neben in-situ-Toncutanen, mit Einschränkungen Alterungsmerkmale an Toncutanen und Silt-Ton-Verfüllungen. Holozän: Nach dem letztmaligen periglazialen Einfluß in der Jüngeren Dryas muß im Holozän weiter Ton verlagert worden sein, da die gut orientierten Toncutane in $\mathrm{Bv}-, \mathrm{A}(\mathrm{e}) \mathrm{l}$ - und $\mathrm{Bt}$ Horizonten sonst nicht zu erklären wären: weitergehende Lessivierung - Hydromorphierung - Verbraunung (Bv-Horizonte)- zuletzt Podsolierung (hier nicht Untersuchungsgegenstand). Hydromorphierung und Verbraunung können auch gleichzeitig unabhängig voneinander in verschiedenen Tiefen ablaufen, bedingt durch die Zweischichtigkeit der Profile. Bei sehr tiefliegenden BtHorizonten (Profil 1) ist dagegen eine hauptsächliche Lessivierung innerhalb der Bt-Horizonte zu vermuten, deren chronologische Einordnung noch aussteht.

Damit wird die schon von KundLeR (1961) getroffene Annahme der Lessivégenese sowohl unter holozänen als auch spätglazialen Milieubedingungen bestätigt und die von RoESCHMANN (1963) vorgeschlagene pedogenetische Prozesschronolo- 

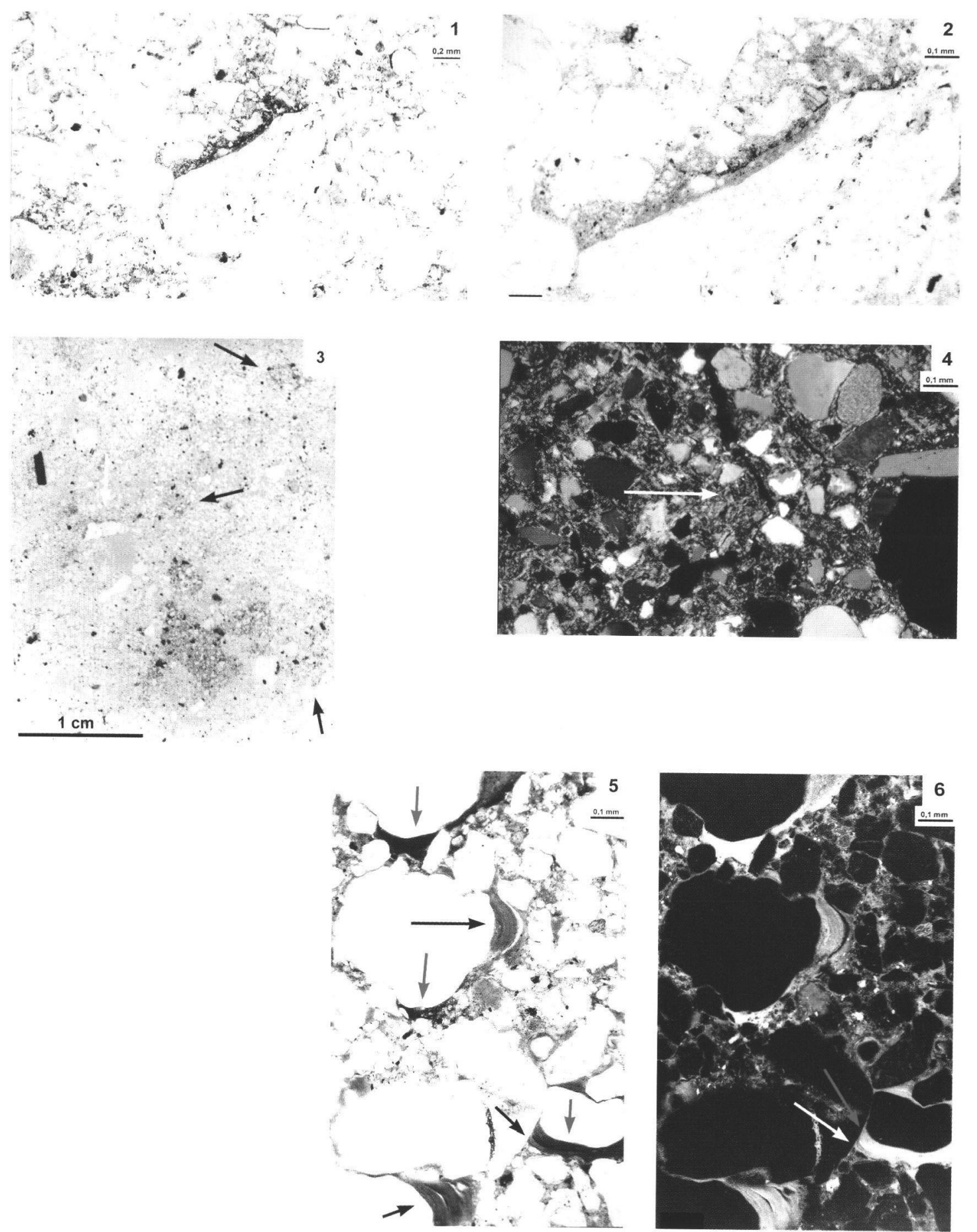
Tafel 3: Dünnschliffbilder von Profil 3; Ael-Horizont: $48 \mathrm{~cm}(1,2)$ und $47 \mathrm{~cm}$ (3) und Ael+Bt-Horizont: $60 \mathrm{~cm}(4), 56 \mathrm{~cm}(5,6)$. (1) Zweiphasige Silt-Ton-Kappe auf Grobsand - Durchlicht (2) Vergrößerung von Photo (2) - Durchlicht. (3) Bt-Schmitzen (schwarze Pfeile) und Silt-Ton-Kappe auf Feinkies (weißer Pfeil) - Dünnschliff. (4) Bt-Schmitze mit Toncutanbruchstück (weißer Pfeil) gekr. Pol.. (5) Staubige dunkelbraune Toncutane (schwarze Pfeile) und sehr staubige (grau)schwarze teilweise silthaltige Toncutane in typischer Position (rote Pfeile); rechts unten: zweiphasiges Toncutane mit innerer sehr staubiger schwarzer Phase - Durchlicht. (6) Inverse Farbgebung verdeutlicht die Mehrphasigkeit der Toncutane (z.B. Pfeile) - wie Photo (5), gekr. Pol.

Plate 3: Photomicrographs of soil profile 3; E-horizon: $47 \mathrm{~cm}(1), 48 \mathrm{~cm}(2,3)$ and E+Bt-horizon $60 \mathrm{~cm}(4), 56 \mathrm{~cm}(5,6)$. (1) Compound silt-clay capping on coarse sand grain - ppl. (2) magnification of photomicrograph (2) - ppl. (3) Bt-streaks (black arrows) and silt clay capping (white arrow) on coarse sand - thin section at $47 \mathrm{~cm}$. (4) Bt-streak with fragment of clay coating (white arrow) - cpl. (5) dusty dark brown clay coatings (black arrows) and very dusty (grey)black clay coatings partly containing silt (red arrows); lower right corner: compound coating with a younger very dusty lamina ppl. (6) inverse colours demonstrate the different phases of clay coatings (cf. arrows) - photomicrograph (5) with cpl.
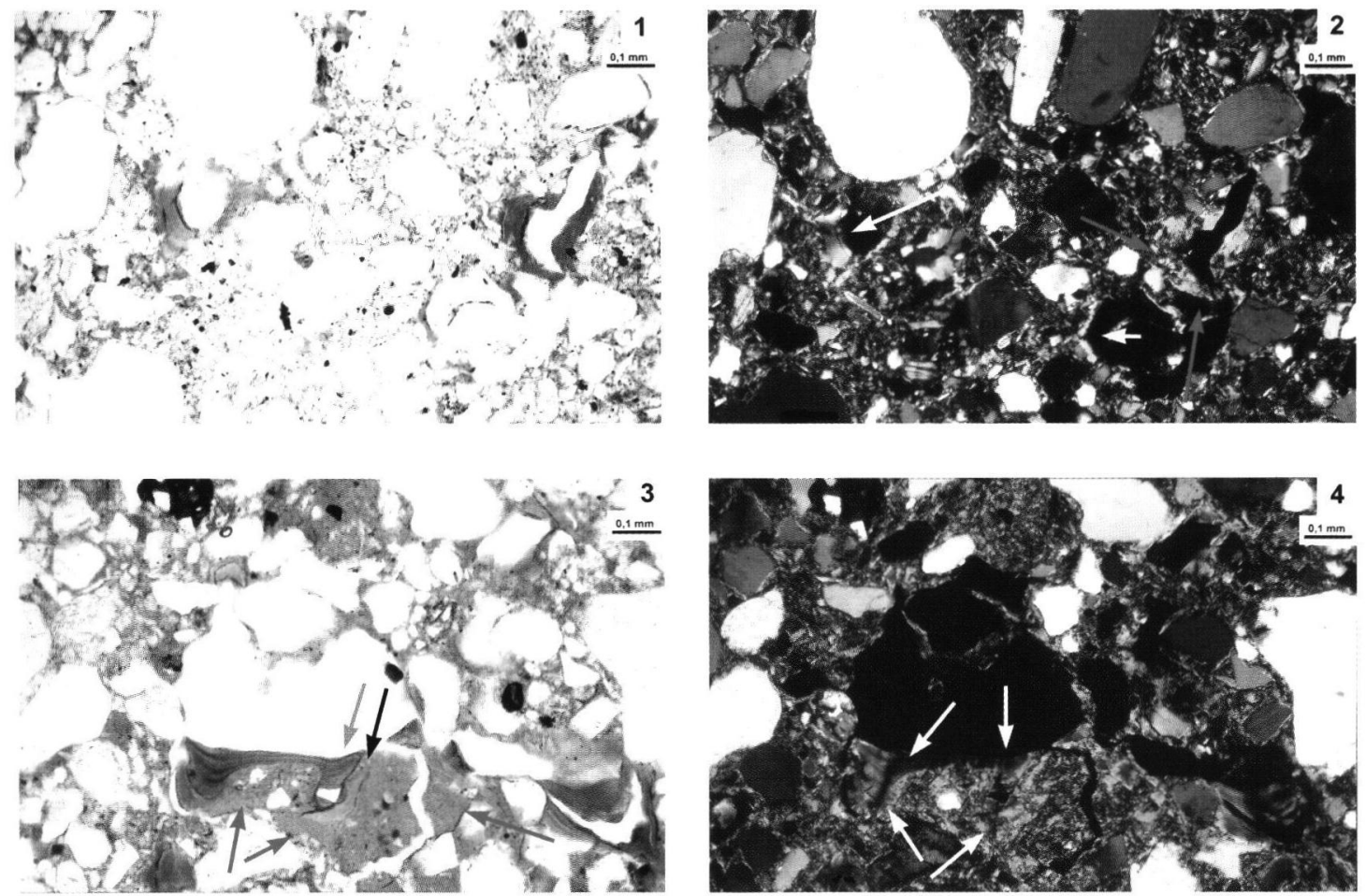

Tafel 4: Dünnschliffbilder von Profil 3; Bt-Horizont $90 \mathrm{~cm}(1,2)$ und $80 \mathrm{~cm}(3,4)$. (1) Photo (2) im Durchlicht. (2) sehr gut orientierte Toncutane mit scharfen Auslöschungsbändern (weiße Pfeile) neben reorientierter (älterer) Toncutane mit undulöser Auslöschung (rote Pfeile) - gekr. Pol. (3) Silt-Ton-Verfüllung (rote Pfeile), in die ein zweiphasiges Toncutane eingelagert ist: Ältere Lamine gelbbraun rein (schwarzer Pfeil); jüngere Lamine dunkelbraun mikrolaminiert (grauer Pfeil) - Durchlicht. (4) sehr gut orientierte Toncutane mit scharfen Auslöschungsbändern (Pfeile), während das undulöse Auslöschungsmuster der Silt-Ton-Verfüllung die Reorientierung des Tons kennzeichnet (Alterungseffekt) - Photo (3) mit gekr. Pol.

Plate 4: Photomicrographs of soil profile 3; Bt-horizon $90 \mathrm{~cm}(1,2)$ and $80 \mathrm{~cm}(3,4)$. (1) Photomicrograph (2) with ppl. (2) Perfectly orientated clay coatings with sharp extinction bands (white arrows) beside a re-orientated (older) clay coating with undulatory extinction (red arrows) - cpl. (3) Silt-clay-infilling (red arrows) with a younger compound clay coating. Older lamina: yellow brown, limpid (black arrow); younger lamina: dark brown with microlamination (grey arrow) - ppl. (4) Sharp extinction bands (arrows) demonstrate the good orientation of the clay coating. Undulatory extinction pattern of the silt-clay-infilling characterizes the reorientation of the clay due to ageing - photomicrograph (3) with cpl. 
gie von Bodenprofilen vergleichbarer Horizontabfolge erweitert.

Dies stimmt mit den Ergebnissen von CATT \& StAINES (1998) aus Südost-England, von KEMP et al. (1998) aus dem nordamerikanischen Jungmoränengebiet und von KÜHN \& KÖSEL (2000) aus dem süddeutschen Jungmoränengebiet überein, die in polygenetischen Bodenprofilen Lessivierung im Spätglazial und Holozän mikromorphologisch nachweisen konnten. Ob der Hauptanteil der Tonverlagerung im Holozän (HoffMANN \& BlumE 1977) stattfand oder im Bölling(!), wie VANVLIETLANOË (1990) anhand mikromorphologischer Untersuchungen an Lessivés im Löß vorschlug, bleibt weiterhin offen.

Humusakkumulation (fAh), schwache Podsolierung (Usselo-Horizont) und schwache Verbraunung (Finow-Boden) sind auf terrestrischen Reinsand-Standorten als im Spätglazial vorkommende pedogenetische Prozesse akzeptiert (VAN GEel et al. 1989, SchlaAK 1998). Auf seit dem Beginn des Spätglazials weitestgehend geomorphodynamisch stabilen Standorten, wie den Grundmoränenplatten des norddeutschen Tieflandes, fand Lessivierung statt, während auf geomorphodynamisch instabilen Standorten (z.B. oben genannte Reinsand-Standorte) Erosion oder Akkumulation die Bodenbildung im Spätglazial immer wieder unterbrachen.

Bestehende Modellvorstellungen zu Bodenentwicklungsreihen im norddeutschen Jungmoränengebiet sind aufgrund der vorgestellten Befunde kritisch zu überprüfen. Bei dem Genesemodell von Reuter (z.B. 1990) beginnt die Bodenbildung erst im Präboreal, während für Kopp (1970) der Hauptanteil der Bodenbildung (Lessivé und Braunerde) im Präboreal weitgehend abgeschlossen ist.

\section{Dank}

Für die finanzielle Unterstützung zur Dünnschliffherstellung der Profile 1 und 2 sei Dr. CoRdsen (LANU Schleswig-Holstein) gedankt. Die Karten und Zeichnungen wurden von P. WIESE und B. LINTZEN angefertigt. Gedankt sei auch den Gutachtern für wertvolle Kritik und Anregungen.

\section{Schriftenverzeichnis}

Altemüller, H.-J. \& Bailly, F. (1976): Mikromorphologische Untersuchungen an einer nordwestdeutschen Parabraunerde-Pseudogley-Sequenz aus Löss. - Geoderma, 16: 327-343; Amsterdam.

Berner, U. \& Streif, H. [Hrsg.] (2000): Klimafakten. 2. verb. Aufl.: 238 S.; Stuttgart (Schweizerbart).
Billwitz, K. (2000): Substrat- und Bodenaufnahme. In: BARSCH, H., Billwitz, K. \& Bork, H. R. [Hrsg.]: Arbeitsmethoden in Physiogeographie und Geoökologie: 172-230; Gotha (Klett-Perthes).

Blume, H.-P. (1968): Zum Mechanismus der Marmorierung und Konkretionsbildung in Stauwasserböden. - Z. Pflanzener., Düng, u. Bodenk., 119: 124 134; Weinheim.

Bronger, A. (1976): Zur quartären Klima- und Landschaftsentwicklung des Karpatenbeckens auf (paläo-)pedologischer und bodengeographischer Grundlage. - Kieler Geogr. Schr., 45: 268 S.; Kiel.

Bullock, P., Fedoroff, N., Jongerius, A., Stoops, G. \& Tursina, T. [Eds.] (1985): Handbook for soil thin section description. - 152 S.; Albrighton, Wolverhampton (Waine Research Publications).

Bullock, P., Milford, M. H. \& Cline, M. G. (1974): Degradation of Argillic Horizons in Udalf Soils of New York State. - Soil Sci. Soc. Am. Proc., 38: 621-628; Madison.

Bussemer, S. (1994): Geomorphologische und bodenkundliche Untersuchungen an periglaziären Deckserien des mittleren und östlichen Barnim. - Berliner Geogr. Arb., 80: 150 S.; Berlin.

Catt, J. A. \& Staines, S. J. (1998): Petrography of sediments and buried soils. - In: PREeCE, R. C. \& BRIDGLAND, D. R. [Eds.]: Late Quaternary Environmental Change in North-west Europe: Excavations at Holywell Coombe, South-east England: 69-85; London (Chapman \& Hall).

De Klerk, P. (2001): Vegetation history and palaeo-environmental development of the Endinger Bruch area and the Reinberg basin (Vorpommern, NE-Germany) during the late Pleniglacial, Lateglacial and Early Holocene (with special emphasis on a wide spread stratigraphic confusion). Inaug.-Diss. Universität Greifswald: 103 S.; Greifswald.

Felix-Henningsen, P. (1979): Merkmale, Genese und Stratigraphie fossiler und reliktischer Bodenbildungen in saalezeitlichen Geschiebelehmen SchleswigHolsteins und Süd-Dänemarks. - Inaug.-Diss. Univ. Kiel: 218 S.; Kiel.

FitzPATRick, E. A. (1993): Soil Microscopy and Micromorphology. - 304 S.; Chichester (Wiley).

GÖRSDORF, J. \& KAISER, K. (2001): Radiokohlenstoffdaten aus dem Spätpleistozän und Frühholozän von Mecklenburg-Vorpommern. - Meyniana, 53: 53-83; Kiel.

Helbig, H. (1999): Die spätglaziale und holozäne Überprägung der Grundmoränenplatten in Vorpommern. - Greifswalder Geogr. Arb., 17: 110 S.; Greifswald.

Hoffmann, R. \& Blume, H. P. (1977): Holozäne Tonverlagerung als profilprägender Prozeß lehmiger Landböden norddeutscher Jungmoränenlandschaften. - Catena, 4: 359-368; Giessen. 
Hujjzer, A. S. (1993): Cryogenic Microfabrics and Macro-structures: Interrelations, Processes and Paleo-eviron-mental Significance. - Thesis Vrije Universiteit Amsterdam: 245 S.; Sassenheim.

ISSS-ISRIC-FAO [Eds.] (1998): World Reference Base for Soil Resources. - World Soil Resources Report, 84 (3): 88 S.; Rome.

JANETZKO, P. (1996): Verbreitung und Gliederung periglaziärer Deckschichten im Jungmoränengebiet von Schleswig-Holstein und ihre Bedeutung für die Pedogenese. - In: LANU Schleswig-Holstein [Hrsg.]: Böden als Zeugen der Landschaftsentwicklung (STREMME-Festschrift): 15-27; Kiel.

JANETZKO, P. \& SсHмIDT, R. (1996): Norddeutsche Jungmoränenlandschaften - Böden als Teile von Landschaften. Kap. 3.4.4.2. - In: Blume et al. [Hrsg.]: Handbuch der Bodenkunde: 36 S.; Landsberg/L. (Ecomed).

Jім, C. Y. (1986): Experimental study of soil microfabrics induced by anisotropic stresses confined swelling and shrinking. - Geoderma, 37: 91-112; Amsterdam.

Jongerius, A. (1970): Some morphological aspects of regrouping phenomena in Dutch soils. - Geoderma, 4: 311-331; Amsterdam.

KAISER, K. F. (1993): Beiträge zur Klimageschichte vom späten Hochglazial bis ins frühe Holozän, rekonstruiert mit Jahrringen und Molluskenschalen. Physische Geographie, 36: 203 S.; Zürich.

Kemp, R. A., McDaniel, P. A. \& Busacca, A. J. (1998): Genesis and relationship of macromorphology and micromorphology to contemporary hydrological conditions of a welded Argixeroll from the Palouse in Idaho. - Geoderma, 83: 309-329; Amsterdam.

Kopp, D. (1970): Periglaziäre Umlagerungs-(Perstruktions) zonen im nordmitteleuropäischen Tiefland und ihre bodengenetische Bedeutung. - Tagungsberichte d. DAL, 102: 55-81; Berlin.

KuBIËNA, W. L. (1986): Grundzüge der Geopedologie und der Formenwandel der Böden. - 128 S.; Wien (Österreichischer Agrarverlag).

KüHN, P. (i. Druck): Grundlegende Voraussetzungen bodengenetischer Vergleichsuntersuchungen: Theorie und Anwendung. - Greifswalder Geogr. Arb., 23; Greifswald.

KÜHN, P. \& KÖSEL, M. (2000): Spätglaziale Boden- und Substratgenese: Mikromorphologisch-stratigraphische Befunde aus dem südwestdeutschen Alpenvorland. - Trierer Bodenkdl. Schr., 1: 51-57; Trier.

KÜHN, P. \& SCHRÖDER, D. (i. Druck): Mikromorphologisch-stratigraphische Befunde zur spätglazialen Bodengenese in NO-Deutschland. - Mitt. dt. bodenkdl. Ges., 96; Oldenburg.

KundLer, P. (1961): Lessivés (Parabraunerden, Fahlerden) aus Geschiebemergel der Würm-Eiszeit im norddeutschen Tiefland. - Z. Pflanzenern., Düng., Bodenk., 95: 97-110; Weinheim.
LAATSCH, W. (1957): Dynamik der mitteleuropäischen Böden. - 4. Aufl.: 280 S.; Dresden, Leipzig (Steinkopff).

LANGOHR, R. (1993): Types of tree windthrow, their impact on the environment and their importance for the understanding of archeological excavation data. - Helinium, 33: 36-49; Wetteren.

LAvES, D. (1972): Beitrag zur Mikromorphologie und Mikro-morpho-genese von Fahlerden (Lessivés). - In: KowA-Linski, S. \& DrozD, J. [Eds.]: Soil micromorphology: 323-335, Proc. 3rd Int. Work. Meet. on Soil Micromorphology, Wroclaw, Poland, 22-28. Sept. 1969; Wroclaw (Panstwowe Wydawnictwo Naukowe).

LAVEs, D. \& ThiERE, J. (1970): Mikromorphologische, chemische und mineralogische Untersuchungen zur Entstehung körnungsdifferenzierter Böden im Jungmoränengebiet der DDR. - Albrecht-ThaerArchiv, 14: 691-699; Berlin.

Macphail, R. (1986): Paleosols in archeology: their role in understanding Flandrian pedogenesis. - In: Wright, V. P. [Ed.]: Paleosols: their recognition and interpretation: 263-290; Princeton, New Jersey.

Meteorologischer und Hydrologischer Dienst der Deutschen Demokratischen Republik [Hrsg.]: Klimatologische Normalwerte für das Gebiet der Deutschen Demokratischen Republik 1901-1950. - 1. u. 2. Lief. $(1955,1961)$; Berlin (Akademie Verlag).

Reuter, G. (1990): Disharmonische Bodenentwicklung auf glaziären Sedimenten unter dem Einfluß der postglazialen Klima- und Vegetationsentwicklung in Mitteleuropa. - In: Ernst-Schlichting-Gedächtniskolloquium, Tagungsband: 69-74; Hohenheim.

Roeschmann, G. (1963): Zur Entstehungsgeschichte von Parabraunerden und Pseudogleyen aus Sandlöß südlich von Bremen. - N. Jb. Geol. Paläo. Abh., 117: 286-302; Stuttgart.

Roeschmann, G. (1968): Besonderheiten der Ton- und Eisenverlagerung in Parabraunerden aus Sandlöß. - Mitt. dt. bodenk. Ges., 8: 277-282; Oldenburg.

Rose, J., Lee, J. A., Kemp, R. A. \& Harding, P. A. (2000): Palaeoclimate, sedimentation and soil development during the Last Glacial Stage (Devensian), Heathrow Airport, London UK. - Quat. Sci. Rev., 19: 827-847; Oxford.

Rúhberg, N., Schulz, W., von Bülow, W., Müller, U., Krienke, H.-D., Bremer, F. \& Dann, T. (1995): Mecklenburg-Vorpommern. - In: BENDA, L. [Hrsg.]: Das Quartär Deutschlands: 95-115; Berlin (Borntraeger).

Russow, F. \& Heinrich, J. (2001): Jungholozäne Überprägungen von quartären Deckschichten und Böden des Mittelgebirgsraumes durch biomechanische Prozesse in Mitteleuropa. - Geoöko, 22: 37 58; Bensheim.

SCHLAAK, N. (1998): Der Finowboden - Zeugnis einer begrabenen weichselspätglazialen Oberfläche in 
den Dünengebieten Nordostbrandenburgs. - Münchener Geogr. Abh., A49: 143-148; München.

Schlichting, E., Blume, H.-P. \& Stahr, K. (1995): Bodenkundliches Praktikum. - 2. neub. Aufl.: 295 S.; Berlin (Parey).

Schneider, R. \& KÜHN, P. (2000): Böden des Karlshofes in Groß Methling, Mecklenburg-Vorpommern. - Trierer Bodenk. Schr., 1: 66-71; Trier.

SCHRÖDER, D. \& SCHNEIDER, R. (1996): Eigenschaften und spätglaziale/holozäne Entwicklung von Böden unterschiedlicher Nutzung aus Decksand über Geschiebemergel in Nord-Ost-Mecklenburg. - In: LANU Schleswig-Holstein [Hrsg.]: Böden als Zeugen der Landschaftsentwicklung (STREMME-Festschrift): 37-47; Kiel.

Schröder, D., Schneider, R. \& KüHn, P. (1997): Entwicklung und Eigenschaften von Böden aus Decksand über Geschiebemergel in NE-Mecklenburg. - Mitt. Dt. Bodenk. Ges., 85: 1243-1246; Oldenburg.

Smith, H. \& WiLding, L. P. (1972): Genesis of Argillic Horizons in Ochraqualfs Derived from Fine Textured Till Deposits of Northwestern Ohio and Southeastern Michigan. - Soil Sci. Soc. Am. Proc., 36: 808-815; Madison.

Smolikova, L. (1968): Mikromorphologie und Mikromorphometrie der pleistozänen Bodenkomplexe. In: Rozpr. Èeskoslov. Akad. Vìd, Øada Matemat. a Pøírod. Vìd, Øoèník 78, Sešit 2: 47 S.; Praha.

Stephan, H.-J. (1995): Schleswig-Holstein. - In: BeNDA, L. [Hrsg.]: Das Quartär Deutschlands: 1-13; Berlin (Born-traeger).

Stephan, S. (2000): Bt-Horizonte als Interglazial-Zeiger in den humiden Mittelbreiten: Bildung, Mikromorphologie, Kriterien. - Eiszeitalter u. Gegenwart, 50: 95-106; Hannover.

STEWIG, R. (1978): Landeskunde von Schleswig-Holstein. - Geocolleg, 5: 216 S.; Kiel (Hirt).

Stoops, G. (1999): Guidelines for Soil Thin Description. Lecture notes prepared for Intensive Course on Soil Micromorphology. 22.3. - 2.4.1999. - ITC Gent: 120 pp.

Tarnocai, C. \& Smith, C. A. S. (1989): Micromorphology and development of some central Yukon paleosols, Canada. - Geoderma, 45: 145-162; Amsterdam.

Van Geel, B., Coope, G. R. \& Van der Hammen, T. (1989): Palaeoecology and stratigraphy of the lateglacial type section at Usselo (The Netherlands). - Rev. Palaeobot. Palynol., 60: 25-129; Amsterdam.

Van Vliet-Lanoë, B. (1985): Frost Effects in Soils. In: BOARDMAN, J. [Ed.]: Soils and Quaternary landscape evolution: 117-158; Chichester (Wiley).

VAn Vliet-Lanoë, B. (1990): The genesis and age of the argillic horizon in Weichselian Loess of Northwestern Europe. - Quat. Intern., 5: 49-56; Oxford.
Van Vliet-Lanoë, B., Fagnart, J. P., Langohr, R. \& Munaut, A. (1992): Importance de la succession des phases écologiques anciennes et actuelles dans la différenciation des sols lessivés de la couverture loessique d'Europe occidentale: argumentation stratigraphique et archéologique. - Science du Sol, 30: 75-93 ;Versailles.

VAN Vliet, B. \& LANGOHR, R. (1981): Correlation between fragipans and permafrost with special reference to silty Weichselian deposits in Belgium and Northern France. - Catena, 8: 137-154; Braunschweig.

VAN Vliet, B. \& LANGohr, R. (1983): Evidence of disturbance of pore ferriargillans in silty soils of Belgium and Northern France. - In: BulLOCK, P. \& MurPHY, C. P. [Eds.]: Soil micromorphology, 2: 515518; Berkhamsted (AB Academic Publishers).

\section{Karten}

Bodenübersichtskarte 1: 200000 (BÜK 200), Blatt CC 2326 Lübeck, Hannover 2001 (i. Druck). 